Document downloaded from:

http://hdl.handle.net/10251/169060

This paper must be cited as:

Sapena-Bano, A.; Martinez-Roman, J.; Puche-Panadero, R.; Pineda Sánchez, M.; PérezCruz, J.; Riera-Guasp, M. (2020). Induction machine model with space harmonics for the diagnosis of rotor eccentricity, based on the convolution theorem. International Journal of Electrical Power \& Energy Systems. 117:1-19. https://doi.org/10.1016/j.ijepes.2019.105625

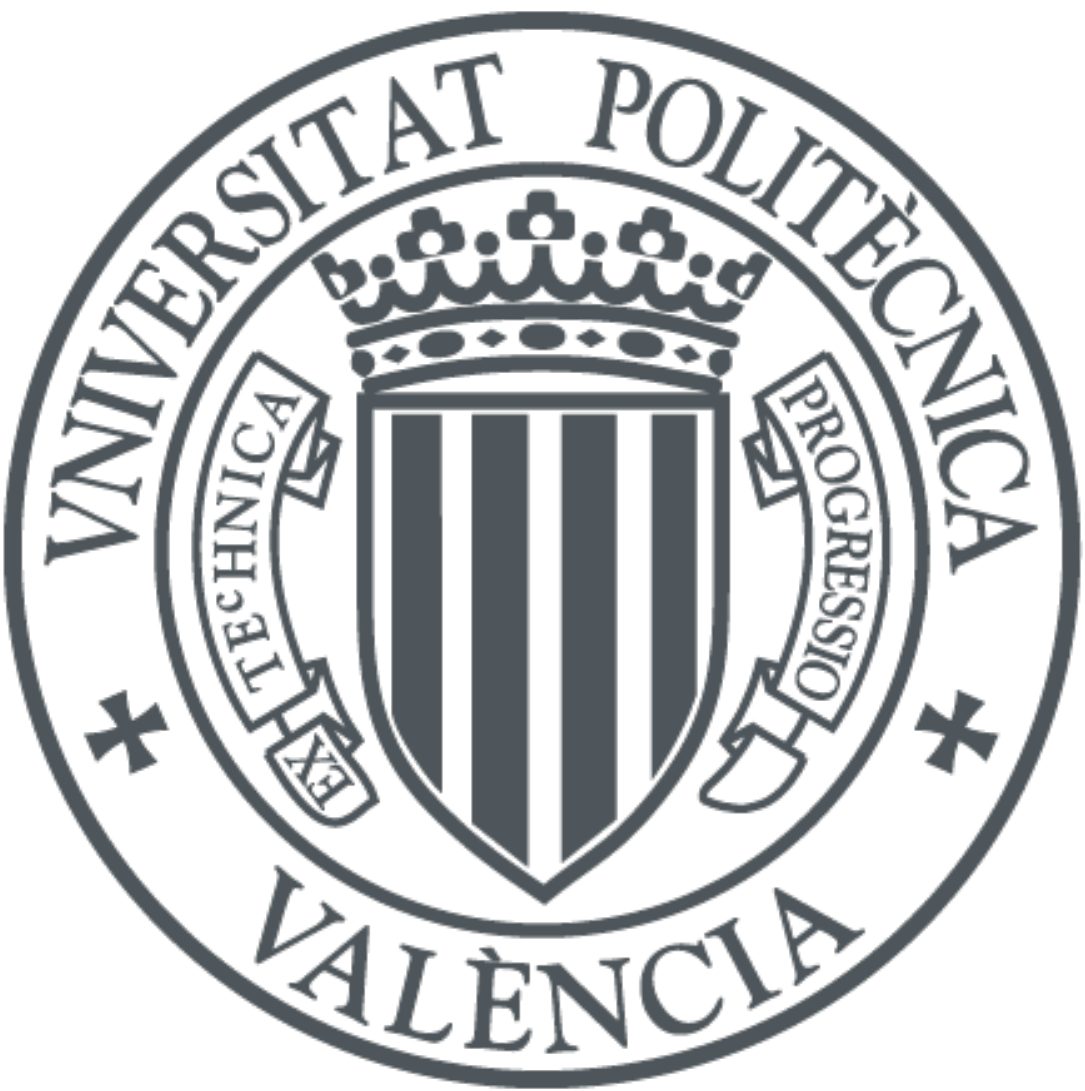

The final publication is available at

https://doi.org/10.1016/j.ijepes.2019.105625

Copyright Elsevier

Additional Information 


\title{
Induction Machine Model with Space Harmonics for the Diagnosis of Rotor Eccentricity, based on the Convolution Theorem
}

\author{
A. Sapena-Bano, J. Martinez-Roman, R. Puche-Panadero*, \\ M. Pineda-Sanchez, J. Perez-Cruz, M. Riera-Guasp \\ Institute for Energy Engineering, Universitat Politècnica de València, Cmno. de Vera \\ $s / n$, 46022 Valencia, Spain
}

\begin{abstract}
Condition based maintenance (CBM) systems of induction machines (IMs) require fast and accurate models that can reproduce the fault related harmonics generated by different kinds of faults. Such models are needed to develop new diagnostic algorithms for detecting the faults at an early stage, to analyse the physical interactions between simultaneous faults of different types, or to train expert systems that can supervise and identify these faults in an autonomous way. To achieve these goals, these models must take into account the space harmonics of the air gap magnetomotive force (MMF) generated by the machine windings under fault conditions, due to the complex interactions between spatial and time harmonics in a faulty machine. One of the most common faults in induction machines is the rotor eccentricity, which can cause significant radial forces and, in extreme cases, produce destructive rotor-stator rub. However, the development of a fast, analytical model of the eccentric IM is a challenging task, due to the non-uniformity of the air gap. In this paper, a new method is proposed to obtain such a fast model. This method, which is theoretically justified, first enables a fast calculation of the self and mutual inductances of the stator and rotor phases for every rotor

\footnotetext{
*Corresponding author

Email addresses: asapena@die.upv.es (A. Sapena-Bano), jmroman@die.upv.es (J. Martinez-Roman), rupucpa@die.upv.es (R. Puche-Panadero), mpineda@die.upv.es (M. Pineda-Sanchez), juperez@die.upv.es (J. Perez-Cruz), mriera@die.upv.es (M. Riera-Guasp)
}

Preprint submitted to INTERNATIONAL JOURNAL OF ELECTRICAL POWER \& ENERGY SYSTEMSMarch 5, 
position, taking into account the non-uniform air-gap length and the actual position of all the stator and rotor conductors. Once these inductances are calculated, they are used in a coupled circuits analytical model of the IM, which in this way is able to calculate the time evolution of the electrical and mechanical quantities that characterize the machine functioning, under any type of eccentricity. Specifically, the model is able to reproduce accurately the characteristic eccentricity fault related harmonics in the spectrum of the stator current.

The proposed approach is validated through two different methods. First, using a finite elements (FEM) model, in order to validate the correctness of the proposed method for calculating self and mutual inductances, taking into account the non-uniform air-gap. Finally, through an experimental test-bed using a commercial induction motor with a forced mixed eccentricity fault, in order to validate that the full model correctly reproduces the phase currents in such a way that their spectra accurately show the harmonics related with the eccentricity fault, which are the basis of many MCSA diagnostic approaches.

Keywords: Inductance, Induction Machines, Convolution, Discrete Fourier Transforms, Fault Diagnosis, Air Gap Eccentricity

\section{Nomenclature}

CBM Condition based maintenance

DE Dynamic eccentricity

FEM Finite elements method

FFT Fast Fourier transform

FRM Field reconstruction method

IFFT Inverse fast Fourier transform

IM Induction machine

MCSA Motor current signature analysis 
ME Mixed eccentricity

MMF Magnetomotive force

MWFA Modified winding function approach

SE Static eccentricity

UMP Unbalanced magnetic pull

\section{Introduction}

Induction motors (IMs) are present in most industrial processes, as driving motors [1] or as generating units [2], due to their inherent robustness and reliability. Nevertheless, they are subject to mechanical and electrical ageing, with the risk of suffering different kinds of faults during their operational life. Their unexpected failure can provoke the sudden stoppage of production lines or power generating stations, with heavy economical losses. CBM systems for IMs [3] can reduce these risks, detecting machine problems prior to a failure [4], and can also help optimize the schedule of maintenance stops. From a broader point of view, CBM systems for IMs can be integrated in maintenance systems for electrical installations, along with CBM systems for inverters [5], generators [6], transformers [7, 8], power systems [9], transmission lines [10] or microgrids [11].

Different signals can be used for implementing a CBM system for IMs, such as currents [12, 13], vibrations [14], instantaneous power [15], voltages [16], thermal images [17], internal flux [18], acoustic emissions [19], etc. Among these methods, the analysis of the machine current signature (MCSA) has attracted a particular interest [20], because it is non invasive, fast and easy to implement online. In its basic version, MCSA needs just a current probe and the fast Fourier transform (FFT) to display the fault signatures in the current spectrum. Besides, it can detect different, (and possibly simultaneous) types of faults [21]. In spite of its simplicity, the practical application of MCSA in harsh industrial environments is a challenging task. The amplitude of the fault harmonics is much smaller than the fundamental component, so that electromagnetic noise produced by electronic converters [22], oscillating loads, or even the FFT spectral leakage can hide the fault harmonics, avoiding their detection until the fault is severe. A fast, analytical model [23] that can reproduce the fault harmonics in the current spectrum, 
under different working conditions, becomes then a valuable resource for the development and testing of robust MCSA algorithms and expert systems for CBM systems [2, 24, 25].

Eccentricity is a common type of IM fault [12, 26-30], which is caused primarily because of maladjustment of bearings, load imbalance, shaft flexibility, thermal deformations, or misalignment [31]. The asymmetry of the magnetic field in the non-uniform air gap of the eccentric IM produces radial forces [30], that is, an unbalanced magnetic pull (UMP) [32-34], which generates abnormal vibrations, damages in the shaft bearings, destructive rotor-stator rub [35] or even sparks during the starting of the motor [36]. Diverse models of the eccentric IM have been proposed recently:

- FEM models: The most accurate models of the eccentric machine are based on the finite elements method (FEM). Transient finite-element models of the eccentric IM have been applied to assess the influence on the UMP of the series/parallel winding connections [37]. A timestepping FEM model is used in [38] to analyse the influence of load variation on the diagnostic indexes of an eccentric motor; in [30] to compute the power balance of an eccentric IM; and in [39] to obtain the characteristic harmonic components generated by an eccentricity fault. Despite its great accuracy, FEM models of an eccentric IM require important computer resources and computing time. To overcome this drawback, an hybrid FEM/superposition approach is proposed in [40], which is able to model an eccentric motor with a saving in time of several orders of magnitude, with a $3 \%$ of relative error compared with a full FEM model. In [41] a 3D field reconstruction method (3D-FRM) is applied to built stator and rotor basis using a reduced number of FEM simulations, maintaining the accuracy of 3D FEM models.

- Analytical models: Diverse analytical models have been presented in the technical literature with an accuracy similar to FEM models, regarding the calculation of fault harmonics, but with a much lower cost. For example, [42] reports a few seconds for the analytical model versus more than three hours for the FEM model, and [43] reports 4 minutes for the analytical model versus 50 hours for the equivalent FEM model. A 3D magnetic equivalent circuit model has been presented in [44], but, in most cases, simpler 2D equivalent circuit models are used for diagnostic purposes. The modified winding function approach (MWFA) [28, 45-49], which uses the inverse air-gap function of the 
eccentric machine to compute the matrix of phase inductances, is a common approach for building such 2D models. But MWFA has some drawbacks: to account for coil pitch, slot skewing or the rise of the air gap MMF across the slot, different winding functions must be used in each case; besides, complex integrals that depend on the winding layout must be solved in this process, which may be very cumbersome in the case of arbitrary winding distributions. As stated in [50], this task typically consumes a high amount of time, so that only discrete curves of inductance versus rotor position are calculated and linear interpolation is applied at intermediate rotor positions. To overcome these difficulties, in $[51,52]$ the convolution theorem has been used to speed-up the calculation of the integrals of the winding functions for eccentric or saturated IMs.

In a previous paper [53], after a critical review of the MWFA, a completely different way of attacking the problem was undertaken, and a new method for computing winding inductances in uniform air gap machines was presented. In the present paper, this method is extended to include the effects of eccentricity, in a two stage process:

- First, a novel analytical expression is derived for representing the yoke flux generated by a single conductor, placed at any angular position in the air-gap, for any rotor position and for any level of eccentricity.

- And, second, a new procedure, based on the spatial convolution of this expression and the distributions of the phases conductors, is developed for obtaining the phase inductances of the eccentric IM, which are used in the equivalent circuit model. This procedure it is very fast, because it is based on the FFT. Besides, the computation time of the FFT of a given winding distribution is practically independent of its complexity.

Once these inductances are calculated, they are introduced in a coupled circuits analytical model of the IM, which in this way is able to reproduce the time evolution of electrical and mechanical quantities of the machine, such as currents, powers, torque speed, etc., with any type of eccentricity and functioning regime; specifically, the proposed model is able to reproduce the characteristic eccentricity fault related harmonics in the stator currents spectra. Thus this model is useful for the development of new eccentricity diagnostic methods based on MCSA techniques. 
The structure of this paper is as follows: in Section 2 the system equations of an induction machine are briefly reminded. In Section 3, the inverse airgap function of the eccentric IM is presented, which is used in Section 4 for deriving the expression of the yoke flux generated by a single conductor in an eccentric IM, as a function of the rotor center. Section 5 establishes the expression of the rotor center coordinates as a function of the rotor angular position, for every type of eccentricity. Based on these results, a new convolution-based method is presented in Section 6 for obtaining the phase inductances for any rotor position, and it is implemented in Section 7 using the FFT. The proposed method for calculating the inductances in eccentric IM is validated in Section 8, where the calculated self and mutual inductances are compared with those obtained through a FEM model; also in this section an experimental validation of the full model is carried out, by comparing the spectra of the simulated current with the spectra of the measured current in a commercial IM with a forced eccentricity fault. Finally, in Section 9, the conclusions of this work are presented.

\section{Induction Machines Electromechanical Equations System}

The following electrical equations system [54,55] can be written for an induction machine with $m$ stator and $n$ rotor phases:

$$
[U]=[R][I]+\frac{d[\Psi]}{d t}=[R][I]+\frac{d([L][I])}{d t}
$$

where $[U]=\left[u_{s 1}, u_{s 2}, \ldots u_{s m}, u_{r 1}, u_{r 2}, \ldots u_{r n}\right]^{T}$ is the phase voltages matrix, $[I]=\left[i_{s 1}, i_{s 2}, \ldots i_{s m}, i_{r 1}, i_{r 2}, \ldots i_{r n}\right]^{T}$ is the phase currents matrix, $[R]$ is the resistances matrix, $[\Psi]$ is the flux linkages matrix and $[L]$ is the inductances matrix. Subscripts $s$ and $r$ are used for the stator and rotor, respectively.

The mechanical equations are:

$$
T_{e}-T_{L}=\frac{1}{2}[I]^{T} \frac{\partial[L]}{\partial \theta}[I]-T_{L}=J \frac{d \Omega}{d t}=J \frac{d^{2} \theta}{d t^{2}}
$$

where $T_{e}$ is the electromechanical torque of the machine, $T_{L}$ is the load torque, $J$ is the total system inertia (rotor plus load), $\Omega$ is the mechanical speed and $\theta$ is the mechanical angle position of the rotor. To solve (1) and (2), the self and mutual phase inductance matrices must be calculated previously for every rotor position. Due to the presence of the derivatives in (1) and (2), it is necessary to achieve a very good accuracy in this process, especially if 
different fault conditions are to be detected and diagnosed in a sure way. The elements of the inductances matrix $[L]$ are computed in this work using a novel approach based on the FFT, taking into account the air gap MMF harmonics and the effect of the rotor eccentricity. End turn and slot leakage inductances need to be pre-calculated, and are included in the $[L]$ terms in (1), as usual in the technical literature, where explicit expressions for these inductances can be found in $[56,57]$.

\section{Modelling of the Eccentric Air Gap Length}

Under the assumption of infinite iron permeability and smooth, constant air gap, the mutual inductances of the phases only change with their relative position. However, for machines with non-uniform air gap, such as the eccentric machines, the value of the air gap length for each angular coordinate at the machine cross section must also be taken into account. If both the stator and rotor cores are cylindrical, the distribution of the air gap lengths at a given time can be completely characterized by the position of the geometric center of the rotor $O_{r}$ with respect to the geometric center of the stator $O_{s}$; and this is true regardless of the type of eccentricity (static, dynamic or mixed eccentricity). To analyse the eccentric machine, a coordinate system attached to the stator will be used in this paper, as shown in Fig. 1 and Fig. 2. In this coordinate system, the effect of eccentricity at every time can be fully characterized by the position of the rotor center (see Fig. 1), as

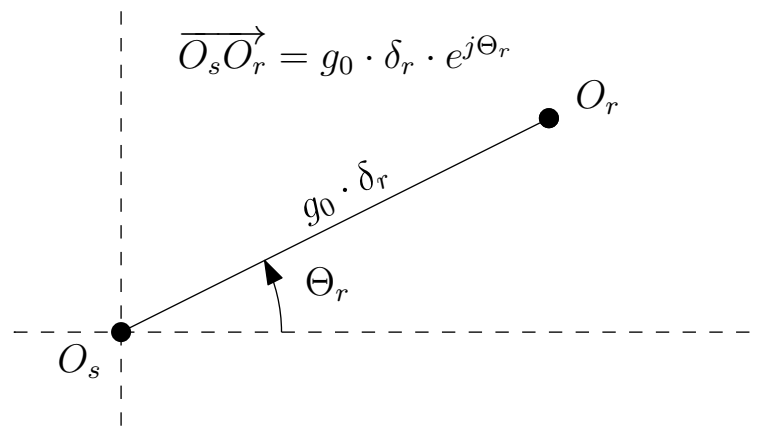

Figure 1: Parameters used for characterizing the level of eccentricity at every time: distance from the rotor center to the stator center $\left(g_{0} \cdot \delta_{r}\right)$, and angular position of the rotor center $\left(\Theta_{r}\right)$, measured in a stator reference frame.

$$
\overrightarrow{O_{s} O_{r}}=g_{0} \cdot \delta_{r} \cdot e^{j \Theta_{r}} \quad 0 \leq \delta_{r}<1,0 \leq \Theta_{r}<2 \pi
$$


where $g_{0}$ is the air gap width of the healthy machine, $\delta_{r}$ is the distance from the rotor axis to the stator axis (in per-unit (p.u.) value of $g_{0}$ ), which is assumed constant along the machine axial length, and $\Theta_{r}$ is the angular position of the rotor center, measured in a stator reference frame.

Assuming, without any loss of generality, that the rotor center lies in the stator $d$-axis, that is, $\Theta_{r}=0$ in Fig. 1, the air gap length at an angular position $\varphi, g(\varphi)$ in Fig. 2, is given by the distance between a point on the external surface of the rotor at this coordinate, $P_{R}$, and a point on the inner surface of the stator at the same coordinate, $P_{S}$. That is,

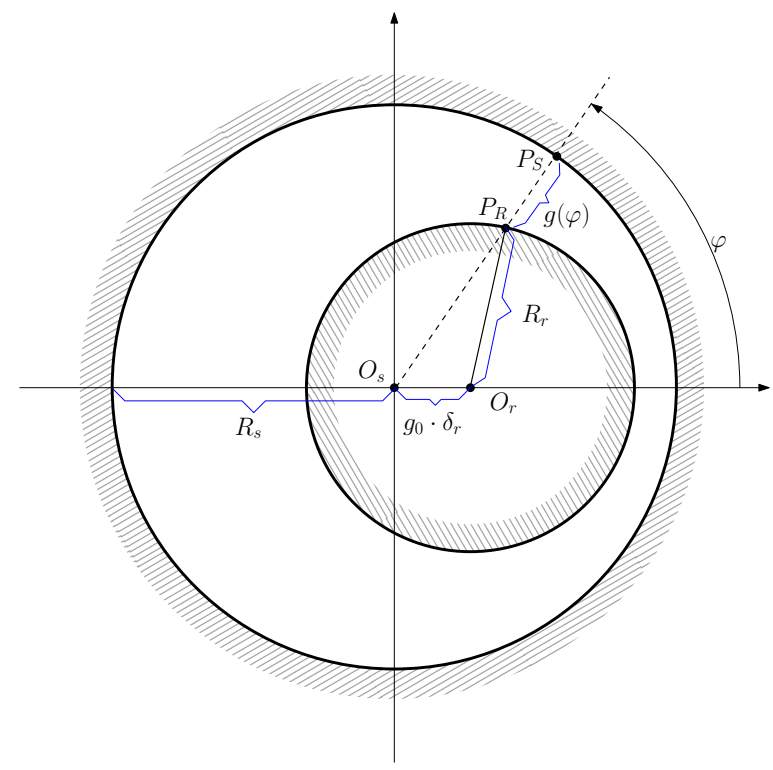

Figure 2: Air gap length $g(\varphi)$ of an eccentric machine as a function of the angular coordinate $\varphi$, measured in a stator reference frame.

$$
g(\varphi)=\left|\overline{O_{s} P_{S}}-\overline{O_{s} P_{R}}\right|=R_{s}-\left|\overline{O_{s} P_{R}}\right|
$$

For computing the phases' inductances, it is needed the inverse of the air gap function to obtain the permeance function of the machine. Based on previous works [58-62], this expression has been obtained in Appendix D as

$$
g\left(\varphi, \Theta_{r}, \delta_{r}\right)^{-1}=g_{0}^{-1} \cdot\left(A_{0}+\sum_{m=1}^{n_{t}} A_{m} \cdot \cos \left(m\left(\varphi-\Theta_{r}\right)\right)\right)
$$


where

$$
A_{0}=\frac{1}{\sqrt{1-\delta_{r}^{2}}} \quad A_{m}=2\left(\frac{1-\sqrt{1-\delta_{r}^{2}}}{\sqrt{1-\delta_{r}^{2}}}\right)^{m} \quad m=1 \ldots n_{t}
$$

It is worth mentioning that only the first term of the series in (5) have been used in [58-61], and two terms in [62]. In this paper, the equations are derived for a generic number $n_{t}$ of terms, where the value of $n_{t}$ can be freely chosen to achieve the desired precision.

\section{Yoke Flux Generated by a Single Conductor in an Eccentric Induction Machine}

Let's consider a conductor of the eccentric induction machine, placed in the air-gap at a given angular position $\alpha$ (Fig. 3).

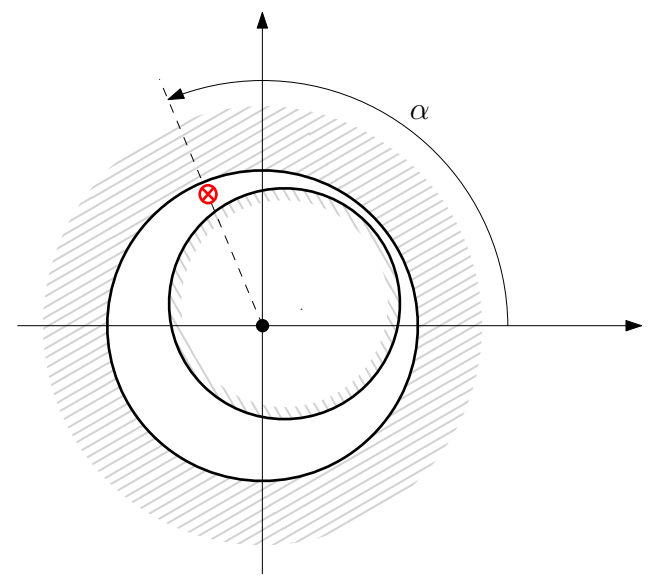

Figure 3: Single conductor of the eccentric induction machine, placed in the air-gap, at a given angular position $\alpha$.

To obtain the yoke flux distribution that this single conductor generates for any given rotor center position $\overrightarrow{O_{s} O_{r}}=\delta_{r} \cdot e^{j \Theta_{r}}$ when it is fed with a unit current, the following steps are taken in this work:

1. The air gap MMF generated by a one-turn, short pitched coil is determined for an eccentric machine, with the rotor center at the arbitrary position $\overrightarrow{O_{s} O_{r}}=\delta_{r} \cdot e^{j \Theta_{r}}$ (Section 4.1). 
2. Based on the air gap MMF of a short pitched coil, the air gap MMF of a single conductor is obtained (Section 4.2).

3. From the air gap MMF of the conductor, the magnetic flux density distribution that it generates along the non-uniform air gap of the eccentric machine is obtained (Section 4.3).

4. Finally, the yoke flux generated by the conductor is calculated, based on its MMF (Section 4.4).

Using the yoke flux of this single conductor in the eccentric machine's air gap, the convolution theorem will be used in Section 6, to obtain the flux linkage of a phase $B$ due to the current flowing through other phase $A$, for any position of the rotor and any relative position of both phases.

\subsection{Air Gap MMF Generated by a One-turn, Short Pitched Coil in an Ec- centric IM}

The air gap MMF generated by a coil along the air gap of an eccentric induction machine at an angular coordinate $\varphi, F_{c}\left(\varphi, \Theta_{r}, \delta_{r}\right)$, is given by

$$
F_{c}\left(\varphi, \Theta_{r}, \delta_{r}\right)=H_{c}\left(\varphi, \Theta_{r}, \delta_{r}\right) \cdot g\left(\varphi, \Theta_{r}, \delta_{r}\right)
$$

where $\varphi$ is the angular coordinate, $\overrightarrow{O_{s} O_{r}}=g_{0} \cdot \delta_{r} \cdot e^{j \Theta_{r}}$ is the position of the rotor geometric center, $H_{c}\left(\varphi, \Theta_{r}, \delta_{r}\right)$ is the mean value of the radial component of the magnetic field intensity at $\varphi$ and $g\left(\varphi, \Theta_{r}, \delta_{r}\right)$ is the air gap length at the angular coordinate $\varphi$.

Let's consider the general case of a short-pitched, one-turn coil, with its first conductor placed at the origin $\varphi=0$, and the other one at position $\varphi=\alpha$ (see Fig. 4a), fed with a unit current, and with the rotor center placed at the arbitrary position $\overrightarrow{O_{s} O_{r}}=g_{0} \cdot \delta_{r} \cdot e^{j \Theta_{r}}$. The air gap MMF generated by this coil at a generic coordinate $\varphi, F_{0 \alpha}\left(\varphi, \Theta_{r}, \delta_{r}\right)$, can be calculated applying Ampere's law to a path as the one labelled 'abcd' in Fig. 4.a, under the assumption of infinite iron permeability, straight conductors and uniform air gap length along the machine axis, in the $z$ direction, as

$$
\begin{cases}F_{0 \alpha}\left(\varphi, \Theta_{r}, \delta_{r}\right)-F_{0 \alpha}\left(0, \Theta_{r}, \delta_{r}\right)=1 & 0 \leq \varphi<\alpha \\ F_{0 \alpha}\left(\varphi, \Theta_{r}, \delta_{r}\right)-F_{0 \alpha}\left(0, \Theta_{r}, \delta_{r}\right)=0 & \alpha \leq \varphi<2 \pi\end{cases}
$$

The total flux crossing a cylindrical surface of radius $r$ and unit length, 
parallel to the stator bore axis, amounts to zero. Therefore

$$
\mu_{0} \cdot r \cdot \int_{0}^{2 \pi} H_{c}\left(\varphi, \Theta_{r}, \delta_{r}\right) \cdot d \varphi=0
$$

and, taking into account (7) and (8), (9) gives

$$
\int_{0}^{\alpha} \frac{\left(1+F_{0 \alpha}\left(0, \Theta_{r}, \delta_{r}\right)\right)}{g\left(\varphi, \Theta_{r}, \delta_{r}\right)} d \varphi+\int_{\alpha}^{2 \pi} \frac{F_{0 \alpha}\left(0, \Theta_{r}, \delta_{r}\right)}{g\left(\varphi, \Theta_{r}, \delta_{r}\right)} d \varphi=0
$$

that is,

$$
F_{0 \alpha}\left(0, \Theta_{r}, \delta_{r}\right)=-\frac{\int_{0}^{\alpha} g\left(\varphi, \Theta_{r}, \delta_{r}\right)^{-1} d \varphi}{\int_{0}^{2 \pi} g\left(\varphi, \Theta_{r}, \delta_{r}\right)^{-1} d \varphi}
$$

Replacing (5) in (11) gives

$$
F_{0 \alpha}\left(0, \Theta_{r}, \delta_{r}\right)=-\frac{\alpha}{2 \pi}-\sum_{m=1}^{n_{t}} \frac{A_{m}}{2 \pi A_{0}} \frac{\sin \left(m \Theta_{r}\right)-\sin \left(m\left(\Theta_{r}-\alpha\right)\right)}{m}
$$

and, combining (12) and (8), gives finally

$$
F_{0 \alpha}\left(\varphi, \Theta_{r}, \delta_{r}\right)=\left\{\begin{array}{cc}
1-\frac{\alpha}{2 \pi}-\sum_{m=1}^{n_{t}} \frac{A_{m}}{2 \pi A_{0}} \frac{\sin \left(m \Theta_{r}\right)-\sin \left(m\left(\Theta_{r}-\alpha\right)\right)}{m} & 0 \leq \varphi<\alpha \\
-\frac{\alpha}{2 \pi}-\sum_{m=1}^{n_{t}} \frac{A_{m}}{2 \pi A_{0}} \frac{\sin \left(m \Theta_{r}\right)-\sin \left(m\left(\Theta_{r}-\alpha\right)\right)}{m} & \alpha \leq \varphi<2 \pi
\end{array}\right.
$$

that is

$$
F_{0 \alpha}\left(\varphi, \Theta_{r}, \delta_{r}\right)=\left\{\begin{array}{rc}
1-\frac{\alpha}{2 \pi}-K_{0 \alpha}\left(\Theta_{r}, \delta_{r}\right) & 0 \leq \varphi<\alpha \\
-\frac{\alpha}{2 \pi}-K_{0 \alpha}\left(\Theta_{r}, \delta_{r}\right) & \alpha \leq \varphi<2 \pi
\end{array}\right.
$$

where

$$
K_{0 \alpha}\left(\Theta_{r}, \delta_{r}\right)=\sum_{m=1}^{n_{t}} \frac{A_{m}}{2 \pi A_{0}} \frac{\sin \left(m \Theta_{r}\right)-\sin \left(m\left(\Theta_{r}-\alpha\right)\right)}{m}
$$

It should be noted that the expression of the MMF generated by a short pitched coil of a healthy (non-eccentric) IM $[53,63]$ can be deduced as a particular case of (14) in which $\delta_{r}=0$ (and, therefore $K_{0 \alpha}\left(\Theta_{r}, \delta_{r}\right)=0$ ); 
Fig. 4.b shows the waves of MMF of the short pitched coil in a healthy machine (red line) and in eccentric one (blue line). It is remarkable that the MMF wave of the eccentric machine can be obtained shifting down the wave of the healthy machine a distance $K_{0 \alpha}$, that only depends on the rotor center position.

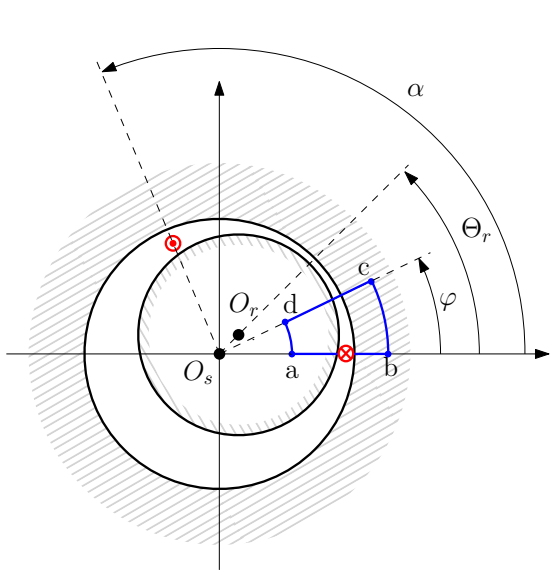

a)

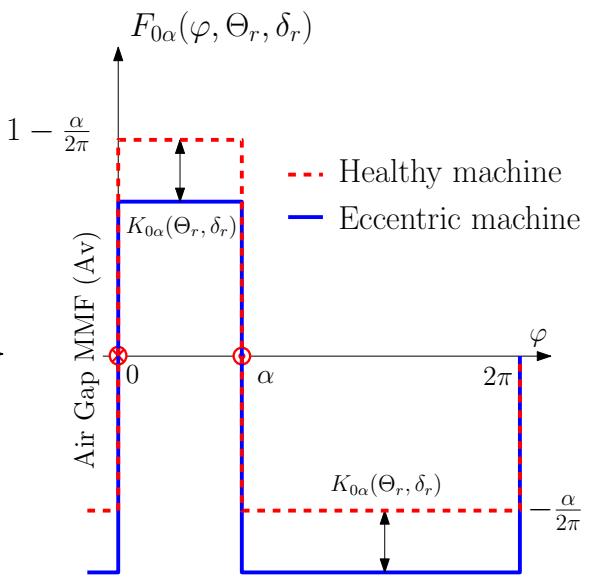

b)

Figure 4: a) Short pitched coil fed by a dc current of 1 A. b) MMF generated by a short pitched coil $F_{0 \alpha}\left(\varphi, \Theta_{r}, \delta_{r}\right)$ in a healthy machine (red, dashed line) and in an eccentric induction machine (blue, solid line), as a function of the angular coordinate $\varphi$, and of the position of the rotor center $\overrightarrow{O_{s} O_{r}}=g_{0} \cdot \delta_{r} \cdot e^{j \Theta_{r}}$.

\subsection{Air Gap MMF Generated by a Single Conductor in an Eccentric IM}

The MMF generated by the short pitched coil (13) can be expressed also as the sum of the MMFs generated by each of its conductors, taking into account the opposite direction of their currents, that is

$$
F_{0 \alpha}\left(\varphi, \Theta_{r}, \delta_{r}\right)=F_{0}\left(\varphi, \Theta_{r}, \delta_{r}\right)-F_{\alpha}\left(\varphi, \Theta_{r}, \delta_{r}\right)
$$

A close inspection of (13) shows the presence of two terms in the summation, each of them corresponding to one of the coil's conductors:

- One of them is proportional to $\sin \left(m \Theta_{r}\right)$, which can be attributed to the MMF of the conductor placed at the origin, $\varphi=0$.

- The other one is proportional to $-\sin \left(m\left(\Theta_{r}-\alpha\right)\right)$, which can be attributed to the MMF of the conductor placed at $\varphi=\alpha$, with the sign reversed to account for the direction of the current. 
Therefore, the expression of the MMF of a single conductor placed at an angular position $\alpha$ in the eccentric machine, $F_{\alpha}\left(\varphi, \Theta_{r}, \delta_{r}\right)$, which satisfies (16), can be expressed as

$$
F_{\alpha}\left(\varphi, \Theta_{r}, \delta_{r}\right)=\left\{\begin{array}{rc}
\frac{1}{2}-\frac{(\varphi-\alpha)}{2 \pi}-K_{\alpha}\left(\Theta_{r}, \delta_{r}\right) & 0 \leq \varphi<\alpha \\
-\frac{1}{2}-\frac{(\varphi-\alpha)}{2 \pi}-K_{\alpha}\left(\Theta_{r}, \delta_{r}\right) & \alpha \leq \varphi<2 \pi
\end{array}\right.
$$

with

$$
K_{\alpha}\left(\Theta_{r}, \delta_{r}\right)=\sum_{m=1}^{n_{t}} \frac{A_{m}}{2 \pi A_{0}} \frac{\sin \left(m\left(\Theta_{r}-\alpha\right)\right)}{m}
$$

Fig. 5.b shows the spatial wave of MMF generated by the single conductor shown in Fig. 5.a. The red line corresponds to the healthy machine, obtained for $\delta_{r}=0, K_{\alpha}\left(\Theta_{r}, \delta_{r}\right)=0$ in (17). The blue line corresponds to an eccentric machine, for which $K_{\alpha}\left(\Theta_{r}, \delta_{r}\right) \neq 0$ in (17). It is noticeable that, similarly to the case of a short pitched coil, the MMF wave generated by a conductor in the eccentric machine can be obtained by shifting down the MMF corresponding to a healthy machine a distance $K_{\alpha}$, which depends on the rotor center position. Furthermore, it should be noted that the expression of the MMF generated by a single conductor of a healthy, non-eccentric IM $[53,63,64]$ can be deduced as a particular case of (17) in which $\delta_{r}=0$ (and, therefore, $K_{\alpha}=0$ ). 


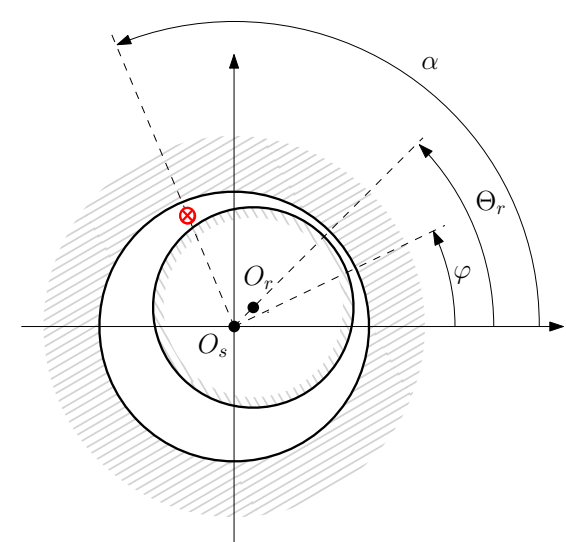

a)

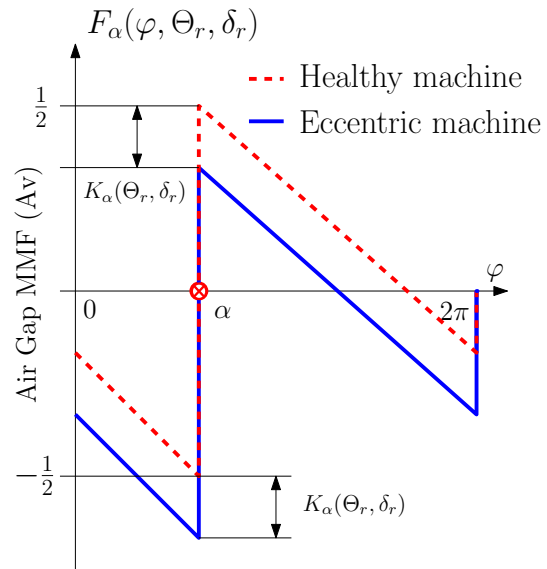

b)

Figure 5: a) Single conductor, placed at an angular position $\alpha$, fed by a dc current of $1 \mathrm{~A}$. b) MMF generated by a single conductor placed at an angular position $\alpha, F_{\alpha}\left(\varphi, \Theta_{r}, \delta_{r}\right)$, in a healthy machine (red, dashed line) and in an eccentric induction machine (blue, solid line), as a function of the angular coordinate $\varphi$, and of the position of the rotor center $\overrightarrow{O_{s} O_{r}}=g_{0} \cdot \delta_{r} \cdot e^{j \Theta_{r}}$.

Eq. (21) can be expressed in a more compact way by wrapping the angular coordinates to the interval $[0,2 \pi)$,

$$
F_{\alpha}\left(\varphi, \Theta_{r}, \delta_{r}\right)=\frac{1}{2}-\frac{((\varphi-\alpha))_{2 \pi}}{2 \pi}-K_{\alpha}\left(\Theta_{r}, \delta_{r}\right)
$$

where $((\varphi-\alpha))_{2 \pi}$ stands for the modulo $2 \pi$ operation

$$
((\varphi-\alpha))_{2 \pi}=\bmod ((\varphi-\alpha+2 \pi), 2 \pi)
$$

For easy of notation, in the rest of this paper the modulo notation will be omitted, and all the angular variables will be assumed to be wrapped to the $[0,2 \pi)$ range, so that (19) will be written as

$$
F_{\alpha}\left(\varphi, \Theta_{r}, \delta_{r}\right)=\frac{1}{2}-\frac{(\varphi-\alpha)}{2 \pi}-K_{\alpha}\left(\Theta_{r}, \delta_{r}\right)
$$

It is worth mentioning that the air gap MMF of an arbitrary coil (e.g. a short-pitched coil) obtained by Ampere's Law, coincides with the one given by summing up the air gap MMFs of its conductors, computed through (21). 
Therefore, the air gap MMF of an arbitrary phase can be expressed as the sum of the air gap MMFs of all of its conductors.

\subsection{Magnetic Flux Density of a Single Conductor in an Eccentric IM}

The radial component of the magnetic flux density, or magnetic induction $B$, at a point of angular coordinate $\varphi$, located at the inner surface of the stator bore, that generates a single conductor placed at an angular position $\alpha$, fed with a unit current, is given by

$$
B_{\alpha}\left(\varphi, \Theta_{r}, \delta_{r}\right)=\mu_{0} \cdot \frac{F_{\alpha}\left(\varphi, \Theta_{r}, \delta_{r}\right)}{g\left(\varphi, \Theta_{r}, \delta_{r}\right)}
$$

and, replacing (5) and (21) in (22), gives

$$
B_{\alpha}\left(\varphi, \Theta_{r}, \delta_{r}\right)=\frac{\mu_{0}}{g_{0}}\left(\frac{1}{2}-\frac{(\varphi-\alpha)}{2 \pi}-K_{\alpha}\left(\Theta_{r}, \delta_{r}\right)\right)\left(A_{0}+\sum_{m=1}^{n_{t}} A_{m} \cos \left(m\left(\varphi-\Theta_{r}\right)\right)\right)
$$

\subsection{Yoke Flux of a Single Conductor in an Eccentric IM}

If the conductor is placed at an angular position $\alpha$ and fed with a unit current, the differential of the magnetic flux due to the conductor which crosses the corresponding air-gap at an angle $\varphi$, for a given position of the rotor center, $\overrightarrow{O_{s} O_{r}}=g_{0} \cdot \delta_{r} \cdot e^{j \Theta_{r}}$, is (Fig. 6)

$$
d\left(\Phi_{\alpha}\left(\varphi, \Theta_{r}, \delta_{r}\right)\right)=\Phi_{\alpha}\left(\varphi+d \varphi, \Theta_{r}, \delta_{r}\right)-\Phi_{\alpha}\left(\varphi, \Theta_{r}, \delta_{r}\right)
$$




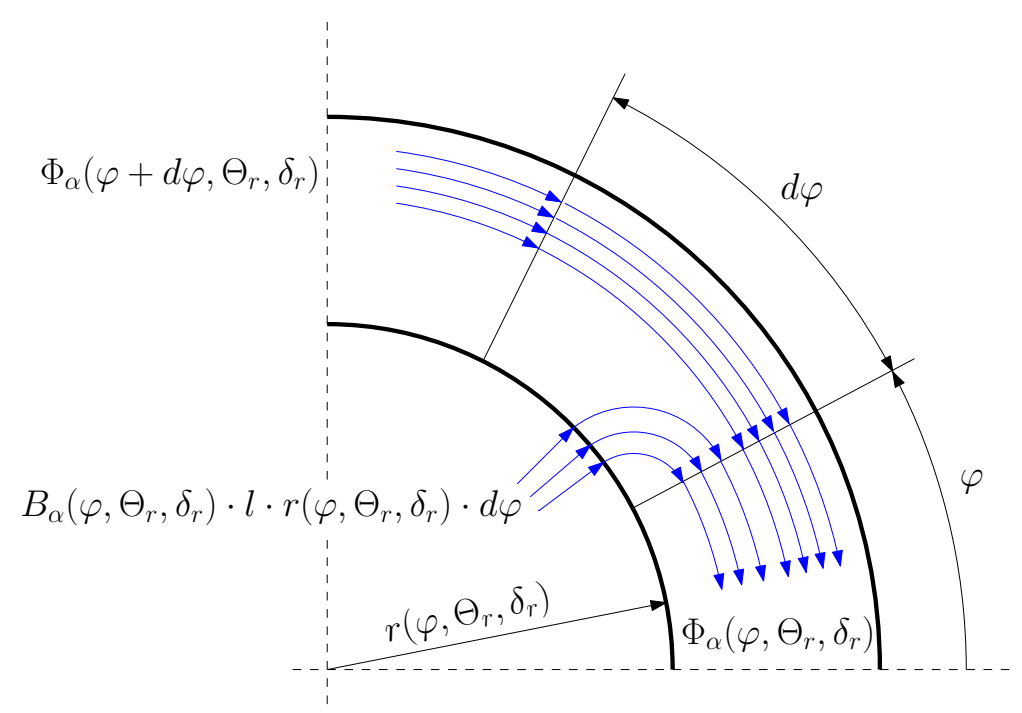

Figure 6: Differential of the yoke flux as a function of the radial component of the induction on the stator inner surface.

The differential of the magnetic flux (24) can be expressed as a function of the induction's radial component [53], as (see Fig. 6)

$$
d\left(\Phi_{\alpha}\left(\varphi, \Theta_{r}, \delta_{r}\right)\right)=-B_{\alpha}\left(\varphi, \Theta_{r}, \delta_{r}\right) \cdot l \cdot r\left(\varphi, \Theta_{r}, \delta_{r}\right) \cdot d \varphi
$$

where $l$ is the axial length of the stator bore. As the air-gap width is considered to be very small, the radius $r\left(\varphi, \Theta_{r}, \delta_{r}\right)$ can be approximated by its mean value $r\left(\varphi, \Theta_{r}, \delta_{r}\right)$, defined in terms of the stator inner radius $R_{s}$ and the rotor's outer radio $R_{r}$ as $[60,61]$

$$
r\left(\varphi, \Theta_{r}, \delta_{r}\right) \approx r=\frac{R_{s}+R_{r}}{2}
$$

The substitution of (26), (22) and (5) in (25) yields

$$
\begin{aligned}
d\left(\Phi_{\alpha}\left(\varphi, \Theta_{r}, \delta_{r}\right)\right)= & -\frac{\mu_{0} l r}{g_{0}}\left(\frac{1}{2}-\frac{(\varphi-\alpha)}{2 \pi}-K_{\alpha}\left(\Theta_{r}, \delta_{r}\right)\right) \cdot \\
& \cdot\left(A_{0}+\sum_{m=1}^{n_{t}} A_{m} \cos \left(m\left(\varphi-\Theta_{r}\right)\right)\right) d \varphi
\end{aligned}
$$


Equation (27) is integrated, which gives

$$
\Phi_{\alpha}\left(\varphi, \Theta_{r}, \delta_{r}\right)=\frac{\mu_{0} l r}{g_{0}} \cdot \Lambda_{\alpha}\left(\varphi, \Theta_{r}, \delta_{r}\right)+C
$$

with

$$
\begin{aligned}
& \Lambda_{\alpha}\left(\varphi, \Theta_{r}, \delta_{r}\right)=\frac{A_{0}}{4 \pi}(\varphi-\alpha)^{2}+\sum_{m=1}^{n_{t}} \frac{A_{m}}{2 \pi}\left(\frac{(\varphi-\alpha) \sin \left(m\left(\varphi-\Theta_{r}\right)\right)}{m}+\frac{\cos \left(m\left(\varphi-\Theta_{r}\right)\right)}{m^{2}}\right)- \\
&\left(\frac{1}{2}-K_{\alpha}\left(\Theta_{r}, \delta_{r}\right)\right) \cdot\left(A_{0}(\varphi-\alpha)+\sum_{m=1}^{n_{t}} A_{m} \frac{\sin \left(m\left(\varphi-\Theta_{r}\right)\right)}{m}\right)
\end{aligned}
$$

The value of constant $C$ in (28) is given by the condition that, due to the cyclic nature of the yoke flux generated by a single conductor, its minimum value is set to zero. Besides, $\Lambda_{\alpha}\left(\varphi, \Theta_{r}, \delta_{r}\right)$ depends only on the level of eccentricity, and is independent of the geometric parameters of the machine. Therefore, it needs to be evaluated only once, and it is scaled to any given machine using the scaling factor $\mu_{0} l r / g_{0}$.

As in the case of the magnetic flux density, the yoke flux generated by an arbitrary phase can be expressed as the sum of the yoke flux generated by all of its conductors.

\section{Position of the Rotor Center as a Function of the Type and Level of Eccentricity}

Eq. (28) gives the yoke flux generated by a single conductor of an eccentric machine as a function of the rotor center coordinates, $\Theta_{r}$ and $\delta_{r}$. But, when the rotor turns around it rotation center by an angle $\theta_{r}(t)$, it is necessary to obtain an expression that gives the coordinates of the rotor center as a function of the rotor angular position $\theta_{r}(t)$. Such an expression is derived in this section, and depends on the type of eccentricity. Three cases will be analysed in this paper: static eccentricity (SE), dynamic eccentricity (DE) and mixed eccentricity (ME). Other types of eccentricity, such as axial [34], inclined [65] or curved eccentricity [33, 46], are outside the scope of this paper. 


\subsection{Static Eccentricity}

$\mathrm{SE}$ is characterized (Fig. 7) by a displacement of the axis of rotation of the rotor $\left(O_{r}\right)$ with respect to the geometric center of the stator $\left(O_{s}\right)$. The axis of rotation of the rotor $O_{\theta}$ coincides with the rotor geometrical center. It can be caused by misalignments of the mounted bearings, or of the bearing plates. The rotor is not centered with the stator bore, but it rotates around its geometric center (30), that is, $\Theta_{r}=$ constant. In the case of static eccentricity, it will assumed in this work, without any of loss of generality, that the rotor center lies in the stator $d$-axis $\left(\Theta_{r}=0\right)$. Therefore, (3) becomes

$$
\overrightarrow{O_{s} O_{r}}=g_{0} \cdot \delta_{r}
$$

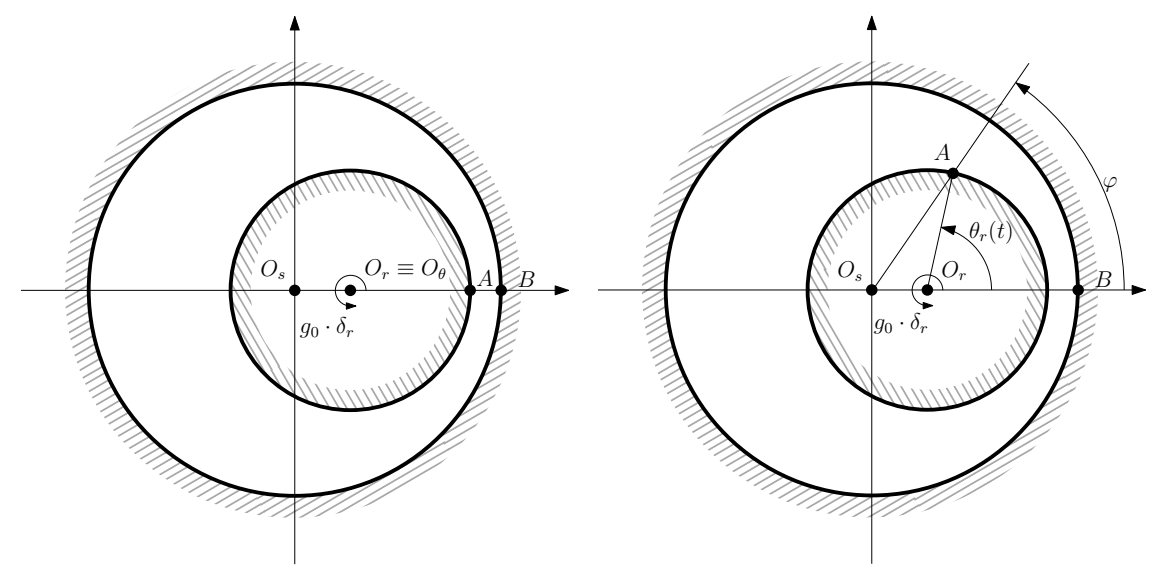

Figure 7: Static eccentricity. Relative position of a rotor conductor, $A$, and a stator conductor, $B$, when the rotor turns an angle $\theta_{r}(t)$ (right) from the initial line (left), in the case of SE. The minimum air gap length is always located at the position of the stator conductor $B$.

The air gap length is non uniform, but its shape does not change when the rotor turns (Fig. 7). Therefore, self and mutual inductances of the stator windings $\left(\mathbf{L}_{\mathbf{s s}}\right)$, are constant, whereas self and mutual inductances of the rotor windings $\left(\mathbf{L}_{\mathbf{r r}}\right)$, and mutual inductances between stator and rotor windings $\left(\mathbf{L}_{\mathbf{s r}}\right)$ change when the rotor turns.

\subsection{Dynamic Eccentricity}

DE is characterized (Fig. 8) by a displacement of the rotor geometric center $\left(O_{r}\right)$ from its rotating axis $\left(O_{\theta}\right)$, which coincides with the stator bore 
axis $\left(O_{s}\right)$. It may be caused by a manufacturing defect, a bent shaft, bearings defects, etc. Under DE, the rotor center spins along a circular path with the same speed as the rotor does. In this case, (3) becomes

$$
\overrightarrow{O_{s} O_{r}}=g_{0} \cdot \delta_{r} \cdot e^{j \theta_{r}}
$$
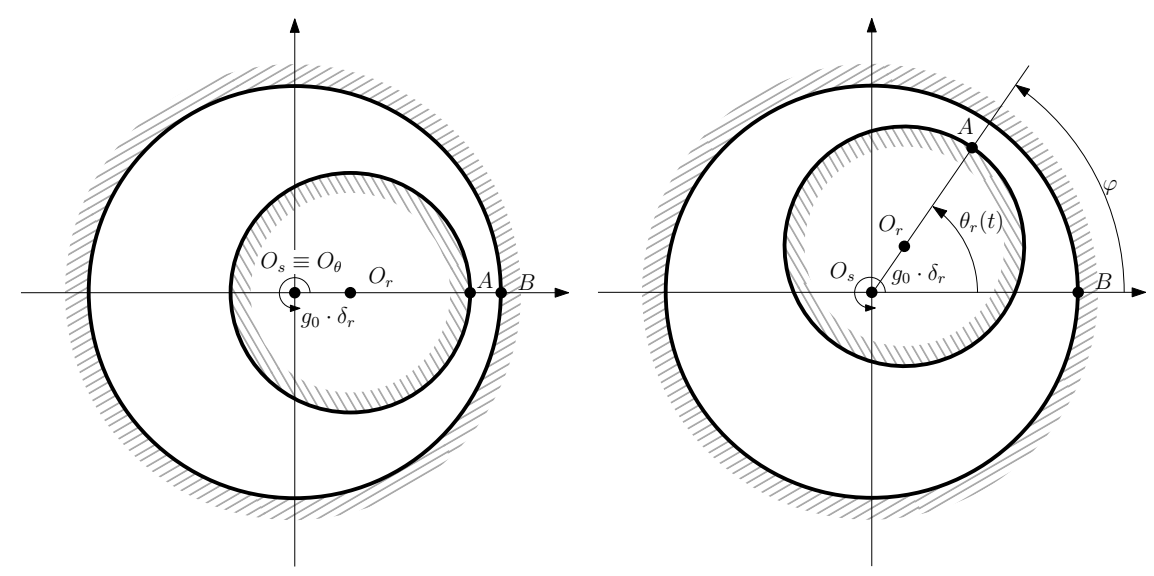

Figure 8: Dynamic eccentricity. Relative position of a rotor conductor, $A$, and a stator conductor, $B$, when the rotor turns an angle $\theta_{r}(t)$ (right) from the initial line (left), in the case of DE. The minimum air-gap length is always located at the position of the rotor conductor $A$.

where $\theta_{r}$ stands for the angle position of the rotor center in stator coordinates. In this case, the position of the minimum air gap rotates with the rotor (Fig. 8). Contrary to the SE case, in DE both $\mathbf{L}_{\mathbf{s s}}$ and $\mathbf{L}_{\mathbf{s r}}$ change when the rotor turns, whereas $\mathbf{L}_{\mathbf{r r}}$ is not affected by the rotation of the machine.

\subsection{Mixed Eccentricity}

$\mathrm{ME}$ appears when both $\mathrm{SE}$ and $\mathrm{DE}$ are present. In this case, the rotating axis $\left(O_{\theta}\right.$ in Fig. 9$)$ is displaced both from the stator geometric center $\left(O_{s}\right)$ and from the rotor center $\left(O_{r}\right)$. From Fig. 9 and (3), the position of the rotor center can be expressed as a function of the level of static eccentricity $\left(\delta_{s e}\right)$ and the level of dynamic eccentricity $\left(\delta_{d e}\right)$ as

$$
\overrightarrow{O_{s} O_{r}}=\overrightarrow{O_{s} O_{\theta}}+\overrightarrow{O_{\theta} O_{r}}=g_{0} \cdot\left(\delta_{s e}+\delta_{d e} \cdot e^{j \theta_{r}}\right)=g_{0} \cdot \delta_{r} \cdot e^{j \Theta_{r}}
$$

with

$$
\delta_{r}=\sqrt{\delta_{s e}^{2}+\delta_{d e}^{2}+2 \cdot \delta_{s e} \cdot \delta_{d e} \cdot \cos \left(\theta_{r}\right)}
$$




$$
\Theta_{r}=\tan ^{-1}\left(\frac{\delta_{d e} \cdot \sin \left(\theta_{r}\right)}{\delta_{s e}+\delta_{d e} \cdot \cos \left(\theta_{r}\right)}\right)
$$

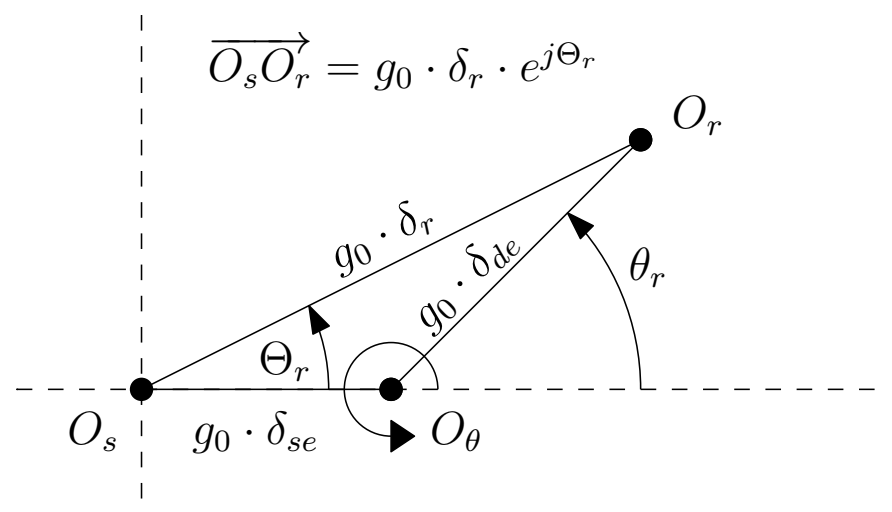

Figure 9: Mixed eccentricity. Position of the rotor center $\left(O_{r}\right)$, the stator center $\left(O_{s}\right)$, and the axis of rotation $\left(O_{\theta}\right)$ in a system of coordinates fixed to the stator, in case of ME.

\subsection{Coordinates of the Rotor Center as a Function of the Type and Level of Eccentricity}

The three types of eccentricity have been defined in the previous sections using the relative position of the rotor center $O_{r}$, the stator center $O_{s}$, and the axis of rotation $O_{\theta}$, as resumed in Table 1 .

Table 1: Definition of the types of rotor eccentricity depending on the relative position of the rotor center $O_{r}$, the stator center $O_{s}$, and the axis of rotation $O_{\theta}$.

\begin{tabular}{cc}
\hline Type & Relationship between $O_{r}, O_{s}$ and $O_{\theta}$ \\
\hline SE & $O_{r}=O_{\theta} \neq O_{s}$ \\
DE & $O_{s}=O_{\theta} \neq O_{r}$ \\
ME & $O_{s} \neq O_{\theta} \neq O_{r}$ \\
\hline
\end{tabular}

The expressions for SE and DE, given by (30) and (31) can be considered as particular cases of (32), as shown in Fig. 10 and in Table 2. 

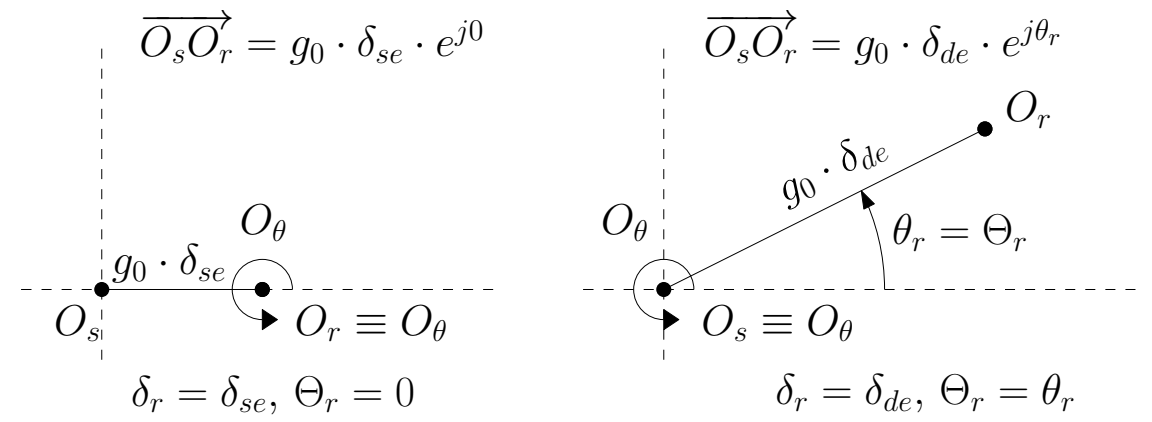

Figure 10: Position of the rotor geometric center $\left(O_{r}\right)$ in a system of coordinates fixed to the stator, in case of static eccentricity (left) and dynamic eccentricity (right).

Table 2: Coordinates of the rotor center for the different types of rotor eccentricity.

\begin{tabular}{ccc}
\hline Type & $\delta_{r}$ & $\Theta_{r}$ \\
\hline SE & $\delta_{s e}$ & 0 \\
DE & $\delta_{d e}$ & $\theta_{r}$ \\
ME & $\sqrt{\delta_{s e}^{2}+\delta_{d e}^{2}+2 \cdot \delta_{s e} \cdot \delta_{d e} \cdot \cos \left(\theta_{r}\right)}$ & $\tan ^{-1}\left(\begin{array}{c}\delta_{d e} \cdot \sin \left(\theta_{r}\right) \\
\delta_{s e}+\delta_{d e} \cdot \cos \left(\theta_{r}\right)\end{array}\right)$ \\
\hline
\end{tabular}

Therefore, the loci of the positions of the geometric rotor center in a reference system fixed to the stator defines the type of eccentricity, as seen in Fig. 11. In the case of a healthy machine (Fig. 11.a), the rotor center is located on the stator geometric center. In the case of SE (Fig. 11.b), the rotor center is placed in a fixed position, different from the stator center. In the case of DE, the rotor center describes a circumference centerd in the stator center (Fig. 11.c). Finally, in the ME case, the rotor center describes a circumference whose center does not coincide with the stator center $\left(\delta_{r} \neq\right.$ const., $\Theta_{r} \neq$ const.). 


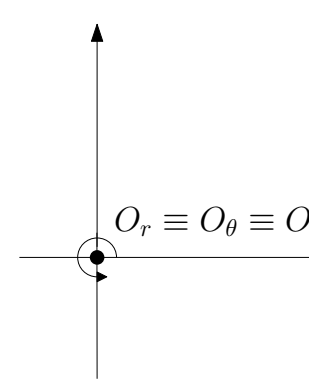

(a)

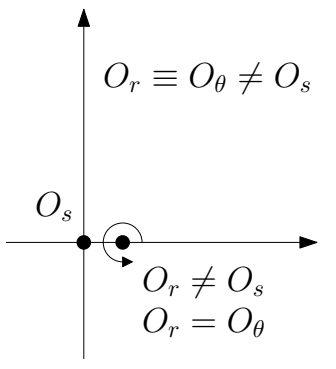

(b)

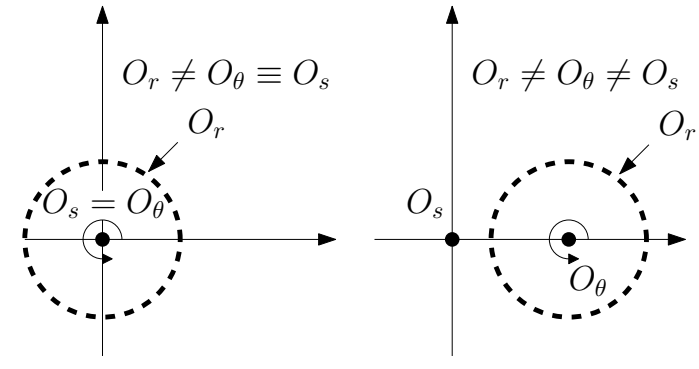

(c)

Figure 11: Loci of the positions of the rotor geometric center $\left(O_{r}\right)$ in a system of coordinates fixed to the stator, in case of (a) healthy machine, (b) static eccentricity, (c) dynamic eccentricity and (d) mixed eccentricity.

In this paper, the most general case, (3), will be analysed, and the result will be applied to each particular type of eccentricity following (32). The election of the position of the rotor center as the variable that characterizes the eccentricity is the key point that enables this unified approach.

\section{Phase Inductances in an Eccentric IM}

For the simulation of an eccentric induction machine with a given level of static $\left(\delta_{s e}\right)$ and dynamic $\left(\delta_{d e}\right)$ eccentricity, it is necessary to obtain the phase inductances matrix for each rotor position, $\theta_{r}$. The goal of this section is to obtain the mutual inductance between two phases of the eccentric machine as a function of their angular positions, for a given position of the rotor. To achieve this goal, it is advisable to express the yoke flux generated by a single conductor as a function of three variables, $\Phi_{\text {cond }}\left(\varphi, \alpha, \theta_{r}\right)$, where $\varphi$ is the angular position where the yoke flux is computed, $\alpha$ is the conductor angular coordinate, and $\theta_{r}$ defines the rotor position. This function can be derived from (28), making use of (33) and (34), as

$$
\Phi_{\text {cond }}\left(\varphi, \alpha, \theta_{r}\right)=\Phi_{\alpha}\left(\varphi, \Theta_{r}\left(\theta_{r}\right), \delta_{r}\left(\theta_{r}\right)\right)=\Phi_{\alpha}\left(\varphi, \theta_{r}\right)
$$

since, for a given level of SE $\left(\delta_{s e}\right)$ and $\mathrm{DE}\left(\delta_{d e}\right)$, the coordinates of the rotor center $\Theta_{r}(34)$ and $\delta_{r}(33)$ depend only on the rotor position $\theta_{r}$ as

$$
\Theta_{r}\left(\theta_{r}\right)=\tan ^{-1}\left(\frac{\delta_{d e} \sin \left(\theta_{r}\right)}{\delta_{s e}+\delta_{d e} \cos \left(\theta_{r}\right)}\right)
$$




$$
\delta_{r}\left(\theta_{r}\right)=\sqrt{\delta_{s e}^{2}+\delta_{d e}^{2}+2 \delta_{s e} \delta_{d e} \cos \left(\theta_{r}\right)}
$$

\subsection{Yoke Flux Generated by a Phase in an Eccentric IM}

Let's consider a phase $A$, with an arbitrary distribution of conductors $Z_{A}(\alpha), 0 \leq \alpha<2 \pi$, where $Z_{A}(\alpha)$ is the number of conductors of phase $A$ on the air gap at angular coordinate $\alpha$. The yoke flux $\Phi_{A}$ that this phase generates when it is fed with a unit current, and shifted a given angle $\varphi_{A}$, can be obtained as a linear superposition of the yoke flux generated by all of the phase's conductors, (35), as

$$
\Phi_{A}\left(\varphi, \varphi_{A}, \theta_{r}\right)=\int_{0}^{2 \pi} \Phi_{c o n d}\left(\varphi, \alpha, \theta_{r}\right) \cdot Z_{A}\left(\alpha-\varphi_{A}\right) \cdot d \alpha
$$

\subsection{Flux Linkages of a Phase in an Eccentric Induction Machine}

Let's consider now a second phase $B$, with an arbitrary distribution of conductors $Z_{B}(\beta), 0 \leq \beta<2 \pi$. The flux linkages of phase $\Psi_{B}$, due to the yoke flux generated by phase $A$, for any given angular position of phases $A$ and $B$, can be obtained just by adding the values of the yoke flux generated by phase $A$ at the yoke sections corresponding to each one of the conductors of phase $B$. Fig. 12 shows the basis of this method: the flux linkage of an arbitrary coil $(a, b)$ can be calculated by replacing the coil by two equivalent annular coils, $\left(a, a^{\prime}\right)$ and $\left(b, b^{\prime}\right)$, and summing up the yoke flux that crosses them, $\Phi\left(\varphi_{a}\right)$ and $\Phi\left(\varphi_{b}\right)$.
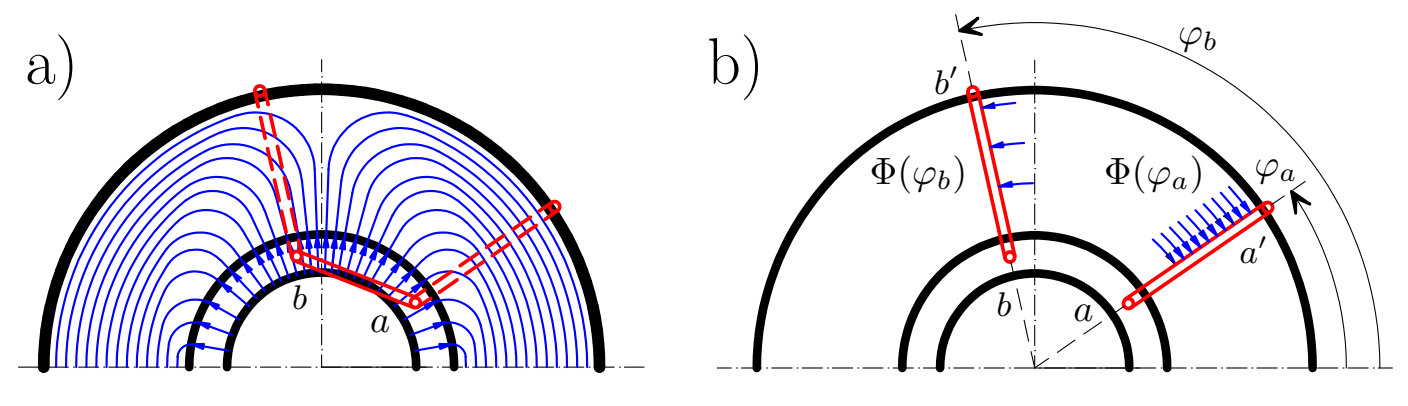

$$
\Psi_{a b}=\Phi\left(\varphi_{a}\right)+\Phi\left(\varphi_{b}\right)
$$

Figure 12: Flux linkage of a single turn coil. a) Actual coil. b) Replaced by two equivalent annular coils.

Following the proposed method, the flux linkages of phase $B$ due to the 
yoke flux generated by phase $A$ are given by

$$
\Psi_{B A}\left(\varphi_{B}, \varphi_{A}, \theta_{r}\right)=\int_{0}^{2 \pi} Z_{B}\left(\beta-\varphi_{B}\right) \cdot \Phi_{A}\left(\varphi, \varphi_{A}, \theta_{r}\right) d \beta
$$

and combining (38) and (39) gives

$\Psi_{B A}\left(\varphi_{B}, \varphi_{A}, \theta_{r}\right)=\int_{0}^{2 \pi} \int_{0}^{2 \pi} Z_{B}\left(\beta-\varphi_{B}\right) \cdot \Phi_{c o n d}\left(\beta, \alpha, \theta_{r}\right) \cdot Z_{A}\left(\alpha-\varphi_{A}\right) \cdot d \alpha d \beta$

As phase $A$ is fed with a unit current, (40) provides the mutual inductance between phases $A$ and $B$ as a function of their angular positions, for a given rotor position

$L_{B A}\left(\varphi_{B}, \varphi_{A}, \theta_{r}\right)=\int_{0}^{2 \pi} \int_{0}^{2 \pi} Z_{B}\left(\beta-\varphi_{B}\right) \cdot \Phi_{c o n d}\left(\beta, \alpha, \theta_{r}\right) \cdot Z_{A}\left(\alpha-\varphi_{A}\right) \cdot d \alpha d \beta$

From (35) and (41) it holds that

$$
L_{B A}\left(\varphi_{B}, \varphi_{A}, \theta_{r}\right)=L_{A B}\left(\varphi_{A}, \varphi_{B}, \theta_{r}\right)
$$

\section{Numerical Computation of the Phase Inductances in an Eccen- tric IM using the FFT}

The computation of (41) can be cumbersome, because a double integral must be computed for obtaining the mutual inductance between two phases $A$ and $B$ for each angular displacement between them, and for all rotor positions. In this section a novel procedure will be applied to simplify this calculation using the FFT. For the numerical computation of (41), the air gap circumference is divided into $N$ equally spaced angular intervals, with a spatial resolution for the angular coordinate $\Delta \varphi=2 \pi / N$. With this discretization, the functions in (41), defined in this discrete mesh, are converted into the following matrices:

$\Phi_{\text {cond }}$ Yoke flux distribution produced by a single conductor $\left(\Phi_{\text {cond }}\left(\varphi, \alpha, \theta_{r}\right) \rightarrow\right.$ $\boldsymbol{\Phi}_{\text {cond }}[i, j, k]$, with dimension $\left.N \times N \times N\right)$.

The element $[i, j, k]$ of the 3D matrix $\boldsymbol{\Phi}_{\text {cond }}$ (43) contains the yoke flux

- at a point of the air gap with an angular coordinate $i \cdot \Delta \varphi$, 
- generated by a single conductor, fed with a unit current, placed at an angular position $j \cdot \Delta \alpha$, with $\Delta \alpha=2 \pi / N$,

- for an angular rotor position equal to $k \cdot \Delta \theta_{r}$, with $\Delta \theta_{r}=2 \pi / N$.

The 3D matrix $\boldsymbol{\Phi}_{\text {cond }}$ is computed, using (35), as

$$
\Phi_{\text {cond }}[i, j, k]=\Phi_{\text {cond }}\left(i \Delta \varphi, j \Delta \alpha, k \Delta \theta_{r}\right) \text { with } i, j, k=0,1, \ldots, N-1
$$

$\mathbf{Z}_{\mathbf{A}}$ Distribution of conductors of phase $A\left(Z_{A}(\alpha) \rightarrow \mathbf{Z}_{\mathbf{A}}[i]\right.$, with dimension $N \times 1$ ) The element [i] of the $1 \mathrm{D}$ matrix $\mathbf{Z}_{\mathbf{A}}(44)$ contains the number of conductors of phase $A$ at an angular position $i \cdot \Delta \varphi$. The position of phase $A$ axis is considered to be aligned with the stator $d$-axis, for fixing a common reference frame in the calculation process.

$$
\mathbf{Z}_{\mathbf{A}}[i]=Z_{A}(i \Delta \varphi) \text { with } i=0,1, \ldots, N-1
$$

$\mathbf{Z}_{\mathbf{B}}$ Distribution of conductors of phase $B,\left(Z_{B}(\beta) \rightarrow \mathbf{Z}_{\mathbf{B}}[i]\right.$, with dimension $N \times 1$ )

$$
\mathbf{Z}_{\mathbf{B}}[i]=Z_{B}(i \Delta \varphi) \text { with } i=0,1, \ldots, N-1
$$

$\mathbf{L}_{\mathbf{B A}}$ Mutual inductance of phases $B$ and $A\left(L_{B A}\left(\varphi_{B}, \varphi_{A}, \theta_{r}\right) \rightarrow \mathbf{L}_{\mathbf{B A}}[i, j, k]\right.$, with dimension $N \times N \times N)$

The element $[i, j, k]$ of the $3 \mathrm{D}$ matrix $\mathbf{L}_{\mathbf{B A}}$ contains the mutual inductance between phases $B$ and $A$ when

- phase $B$ is placed at position $i \cdot \Delta \varphi_{B}$, with $\Delta \varphi_{B}=2 \pi / N$,

- phase $A$ is placed at position $j \cdot \Delta \varphi_{A}$, with $\Delta \varphi_{A}=2 \pi / N$,

- the rotor center is placed at an angular position $k \cdot \Delta \theta_{r}$.

These four matrices have been represented in Fig. 13. 

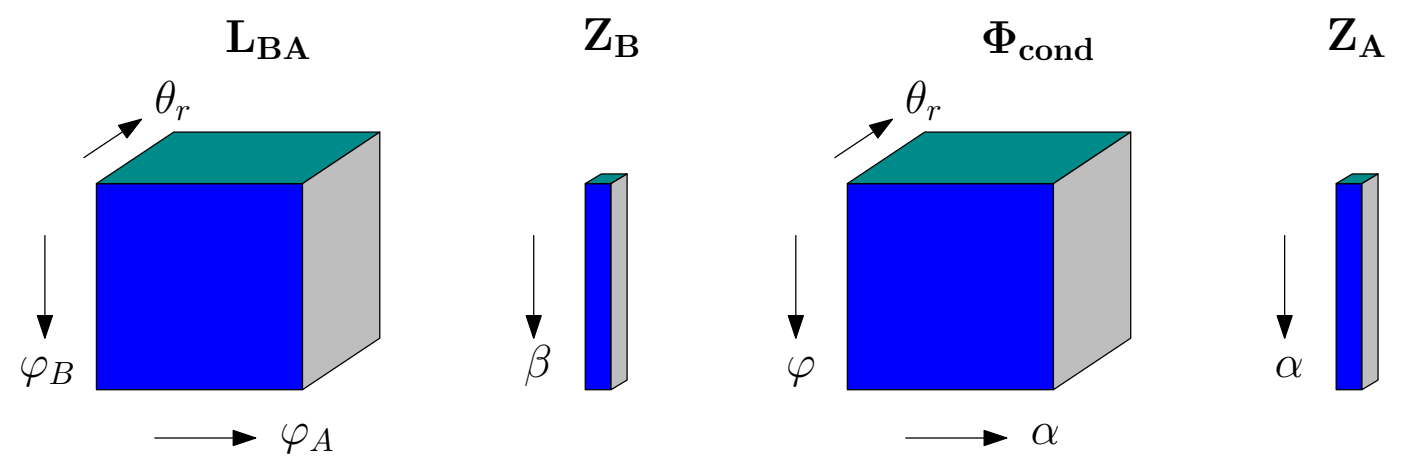

Figure 13: Matrices used for computing the mutual inductance between phases $B$ and $A$, $\mathbf{L}_{\mathbf{B A}}$, for every angular position of phase $B, \varphi_{B}$, of phase $A, \varphi_{A}$, and of the rotor $\theta_{r}$.

The 3D matrix $\mathbf{L}_{\mathbf{B A}}$ can be computed in a very simple and effective way, making use of the properties of the FFT, and its inverse (IFFT), as

$$
\mathbf{L}_{\mathbf{B A}}=\operatorname{IFFT}\left\{\left(\left(\operatorname{FFT}\left\{\mathbf{Z}_{\mathbf{A}}\right\}\right)^{\prime} * \operatorname{FFT}\left\{\boldsymbol{\Phi}_{\text {cond }}\right\} * \operatorname{FFT}\left\{\mathbf{Z}_{\mathbf{B}}\right\}\right)\right\}
$$

where the symbol' stands for the non-conjugate matrix transpose transformation, and the symbol * stands for an element-by-element row or column multiplication. That is, each column of the FFT of $\boldsymbol{\Phi}_{\text {cond }}$ is multiplied element-by-element by the FFT of $\mathbf{Z}_{\mathbf{B}}$, and each row of the resulting matrix is multiplied element-by-element by the transposed FFT of $\mathbf{Z}_{\mathbf{A}}$. The inverse FFT of this product gives directly the inductances matrix $\mathbf{L}_{\mathbf{B A}}$.

Equation (46) is based on the convolution theorem, which states that the FT of the convolution of two functions is equal to the product of their FTs. In [53] this theorem was applied to the computation of phase inductances, using 1D matrices for representing the phase conductor distributions and the yoke flux generated by a single conductor. Equation (46) is an extension of this procedure to the case of an eccentric machine, where the yoke flux generated by a conductor must be represented using a full 3D matrix $\boldsymbol{\Phi}_{\text {cond }}$, to take into account the influence of the rotor center position. Moreover, it can be programmed very easily in commercial software packages. For example, in MATLAB language it is simply written as

$$
\mathrm{LBA}=\operatorname{ifftn}\left(\left(\operatorname{fft}(\mathrm{ZA}) .^{\prime} * * f f \operatorname{tn}(\text { PhyCond }) \cdot * \operatorname{fft}(\mathrm{ZB})\right)\right)
$$

For a given IM, it is necessary to compute the inductance matrices $\mathbf{L}_{\mathbf{s s}}$, $\mathbf{L}_{\mathbf{r r}}, \mathbf{L}_{\mathbf{s r}}$ and $\mathbf{L}_{\mathbf{r s}}$, which are used in eqs (1) and (2). Denoting $\mathbf{Z}_{\mathbf{s}}$ as the 
distribution of the conductors or a stator phase (assuming that all the stator phases are identical), and $\mathbf{Z}_{\mathbf{r}}$ as the distribution of the conductors or a rotor phase (assuming also that all the rotor phases are identical), (46) must be particularized for the following cases:

- Phases $A$ and $B$ in the stator $\left(\mathbf{Z}_{\mathbf{A}}=\mathbf{Z}_{\mathbf{B}}=\mathbf{Z}_{\mathbf{s}}\right)$

$$
\mathbf{L}_{\mathbf{s s}}=\operatorname{IFFT}\left\{\left(\left(\operatorname{FFT}\left\{\mathbf{Z}_{\mathbf{s}}\right\}\right)^{\prime} * \operatorname{FFT}\left\{\boldsymbol{\Phi}_{\text {cond }}\right\} * \operatorname{FFT}\left\{\mathbf{Z}_{\mathbf{s}}\right\}\right)\right\}
$$

- Phases $A$ and $B$ in the rotor $\left(\mathbf{Z}_{\mathbf{A}}=\mathbf{Z}_{\mathbf{B}}=\mathbf{Z}_{\mathbf{r}}\right)$

$$
\mathbf{L}_{\mathbf{r r}}=\operatorname{IFFT}\left\{\left(\left(\operatorname{FFT}\left\{\mathbf{Z}_{\mathbf{r}}\right\}\right)^{\prime} * \operatorname{FFT}\left\{\boldsymbol{\Phi}_{\text {cond }}\right\} * \operatorname{FFT}\left\{\mathbf{Z}_{\mathbf{r}}\right\}\right)\right\}
$$

- Phase $A$ in the stator $\left(\mathbf{Z}_{\mathbf{A}}=\mathbf{Z}_{\mathbf{s}}\right)$ and phase $B$ in the rotor $\left(\mathbf{Z}_{\mathbf{B}}=\mathbf{Z}_{\mathbf{r}}\right)$

$$
\mathbf{L}_{\mathbf{s r}}=\operatorname{IFFT}\left\{\left(\left(\operatorname{FFT}\left\{\mathbf{Z}_{\mathbf{s}}\right\}\right)^{\prime} * \operatorname{FFT}\left\{\boldsymbol{\Phi}_{\text {cond }}\right\} * \operatorname{FFT}\left\{\mathbf{Z}_{\mathbf{r}}\right\}\right)\right\}
$$

- Phase $A$ in the rotor $\left(\mathbf{Z}_{\mathbf{A}}=\mathbf{Z}_{\mathbf{r}}\right)$ and phase $B$ in the stator $\left(\mathbf{Z}_{\mathbf{B}}=\mathbf{Z}_{\mathbf{s}}\right)$

$$
\mathbf{L}_{\mathbf{r s}}=\operatorname{IFFT}\left\{\left(\left(\operatorname{FFT}\left\{\mathbf{Z}_{\mathbf{r}}\right\}\right)^{\prime} * \operatorname{FFT}\left\{\boldsymbol{\Phi}_{\text {cond }}\right\} * \operatorname{FFT}\left\{\mathbf{Z}_{\mathbf{s}}\right\}\right)\right\}
$$

It is worth mentioning that, in equations (48), (49), (50) and (51), the term $\boldsymbol{\Phi}_{\text {cond }}$ is the same. Therefore, it must be computed just once. Moreover, this term is valid for all IM machines with the same level of eccentricity, except from a scale factor $\frac{\mu_{0} l r}{g_{0}}(28)$. Besides, $\mathbf{L}_{\mathbf{r s}}=\mathbf{L}_{\mathbf{s r}}{ }^{\prime}$, which makes unnecessary to compute (51).

As for the computation time for equations (48), (49), (50) and (51), the proposed method, being based on the FFT, is very fast, and practically independent from the complexity of the winding distributions. In this work it has been applied to the motor whose characteristics are given in Appendix A, with a value of $N=1008$. The time needed with the computer of Appendix $\mathrm{C}$ is 0.00045 seconds to obtain the vector $\operatorname{FFT}\left\{\mathbf{Z}_{\mathbf{s}}\right\}$, and 0.00074 seconds to obtain the vector FFT $\left\{\mathbf{Z}_{\mathbf{r}}\right\}$. Therefore, the impact of the complexity of the phase winding is negligible. The computation of the FFT of the $3 \mathrm{D}$ matrix $\boldsymbol{\Phi}_{\text {cond }}$ takes a longer time, 604 seconds. 
The distribution of the phase conductors $\mathbf{Z}_{\mathbf{A}}$ in (44) and $\mathbf{Z}_{\mathbf{B}}$ in (45) have been assumed aligned with the d-axis, for simplicity of (46). If this condition is not met, by choosing other origin of angular coordinates, and the distribution of conductors is not symmetric with respect to this new origin, then $\operatorname{FFT}\left\{\mathbf{Z}_{\mathbf{A}}\right\}$ and $\operatorname{FFT}\left\{\mathbf{Z}_{\mathbf{B}}\right\}$ in (46) must be replaced by their conjugates, that is, $\operatorname{conj}\left(\operatorname{FFT}\left\{\mathbf{Z}_{\mathbf{A}}\right\}\right)$ and $\operatorname{conj}\left(\operatorname{FFT}\left\{\mathbf{Z}_{\mathbf{B}}\right\}\right)$.

\section{Numerical and Experimental Validation}

The proposed method has been validated, both numerically and experimentally, using a commercial IM (whose characteristics are given in Appendix A) with a provoked mixed eccentricity fault, characterized by $\delta_{s e}=0.3$ and $\delta_{d e}=0.3$.

\subsection{Numerical Validation}

For the numerical validation of the proposed method, a finite element model (FEM) of the motor has been implemented using FEMM software [66]. For this simulation, a value of $N=1008$ rotor positions has been selected, obtained by multiplying the rotor and the stator number of slots. For each rotor position, one of the machine phases is fed with an unit current, and the flux linkages of all the phases are computed, giving their mutual inductances. The same procedure is repeated for all the IM phases, and for all rotor positions, giving a total number of simulations equal to $31 \times 1008=31248$ simulations, with a total time of 1300 hours, using the computer of Appendix C. Fig. 14 shows the simulations for a rotor position at the origin, for the first phase of the stator (Fig. 14, top) and of the first rotor loop, constituted by two adjacent bars and their end-ring connections (Fig. 14, bottom). 

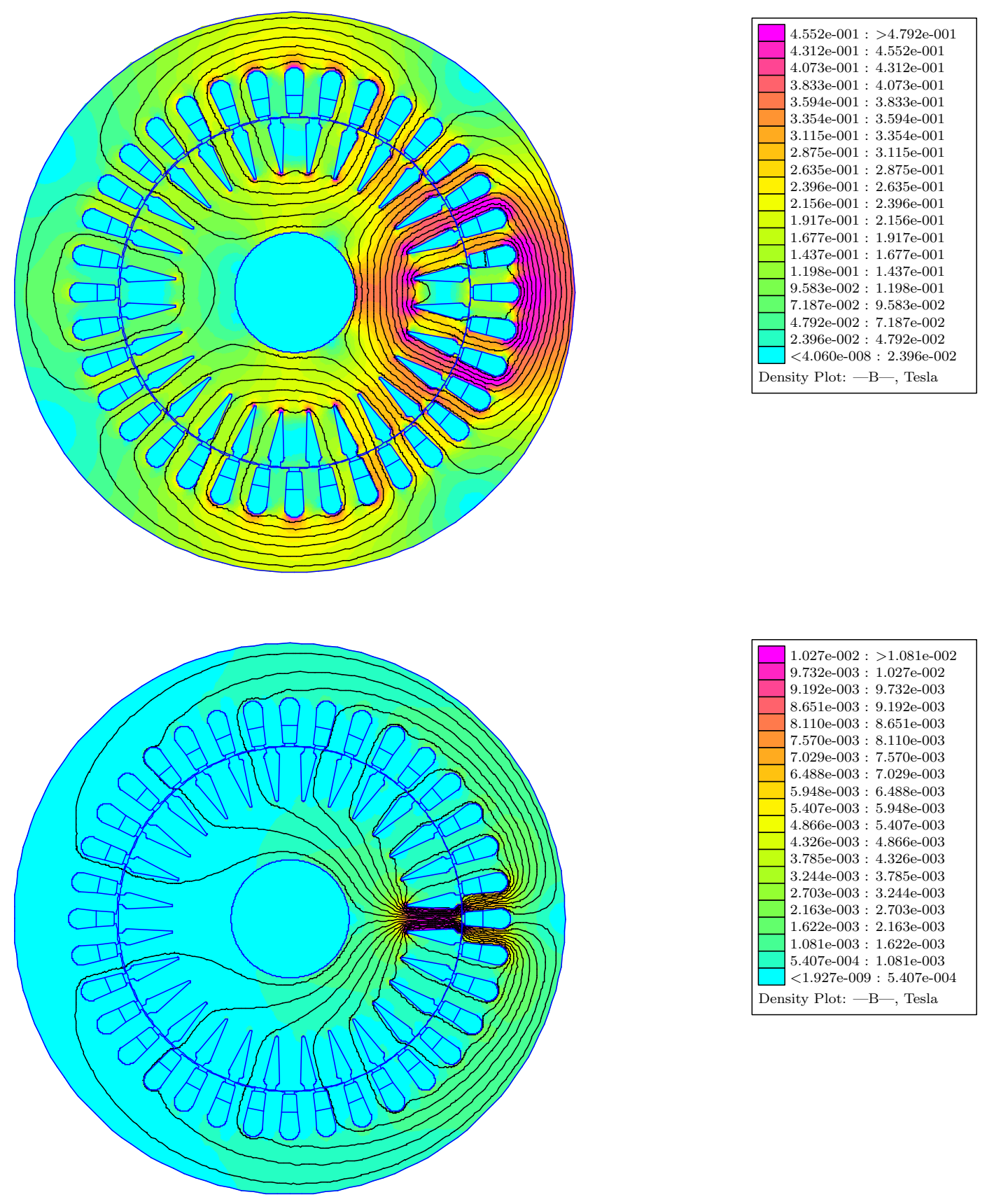

Figure 14: FEM simulation of the IM of Appendix A with an eccentricity level of $\delta_{s e}=$ 0.3 and $\delta_{d e}=0.3$. Top: first stator phase fed with a unit current. Bottom: first rotor loop fed with a unit current. 
The same machine has been simulated with the method proposed in this paper. The analytical solution, which gives the mutual inductances between all the phases for the $N=1008$ different angular rotor positions, has been obtained in just 10 minutes, using the same computer of Appendix C. Fig. 15 compares the inductances calculated for 1008 different rotor positions, using the FEM, and the proposed analytical method. In both cases, the machine of Appendix A is used, with a mixed eccentricity characterized by $\delta_{s e}=0.3$ and $\delta_{d e}=0.3$. Fig. 15, top, compares the mutual inductance between the first stator phase and the first rotor loop for different positions of the rotor. Fig. 15, middle, compares the self inductance of the first rotor loop for different positions of the rotor. Finally, Fig. 15, bottom, compares the self inductance of the first stator phase for different positions of the rotor. A good agreement is observed in the three comparisons of Fig. 15. The analytical model, unlike the FEM model, does not take into account the influence of slotting, but, except this difference, the changes of the inductances produced by the rotor position are very similar with both models.

The same comparison presented in Fig. 15 has been carried out for two extreme cases: a pure static eccentricity fault, presented in Fig. 16, and a pure dynamic eccentricity fault, shown in Fig. 17. As in the case of mixed eccentricity fault, presented in Fig. 15, the agreement between the inductances computed using the FEM model and the analytical model are very good. 

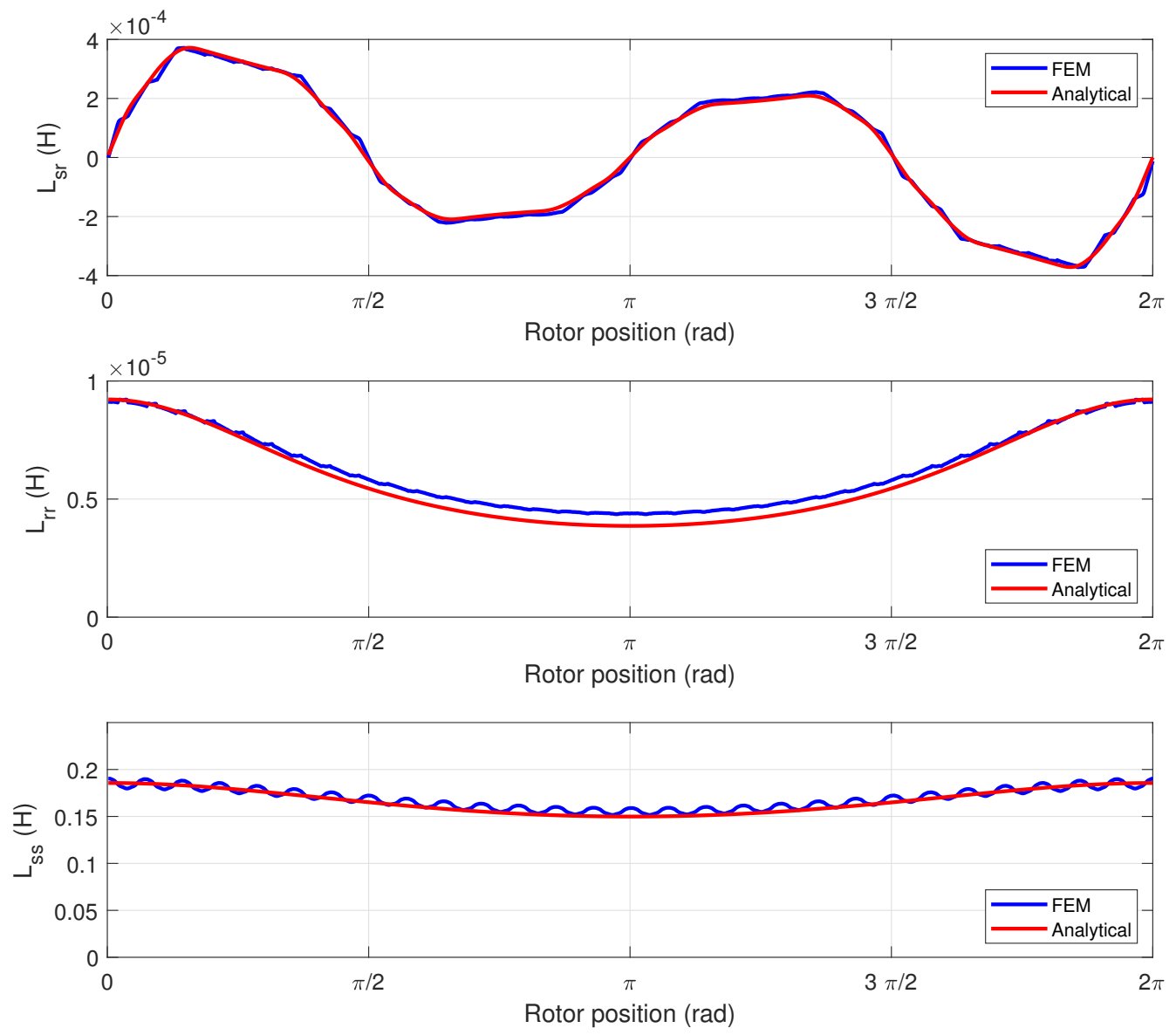

Figure 15: Comparison between the inductances obtained via FEM simulation (blue line) and with the proposed analytical method (red line), for the IM of Appendix A with an eccentricity level of $\delta_{s e}=0.3$ and $\delta_{d e}=0.3$ (mixed eccentricity). Top: mutual inductance between the first stator phase and the first rotor loop. Middle: self-inductance of the first rotor loop. Bottom: self-inductance of the first stator phase. 

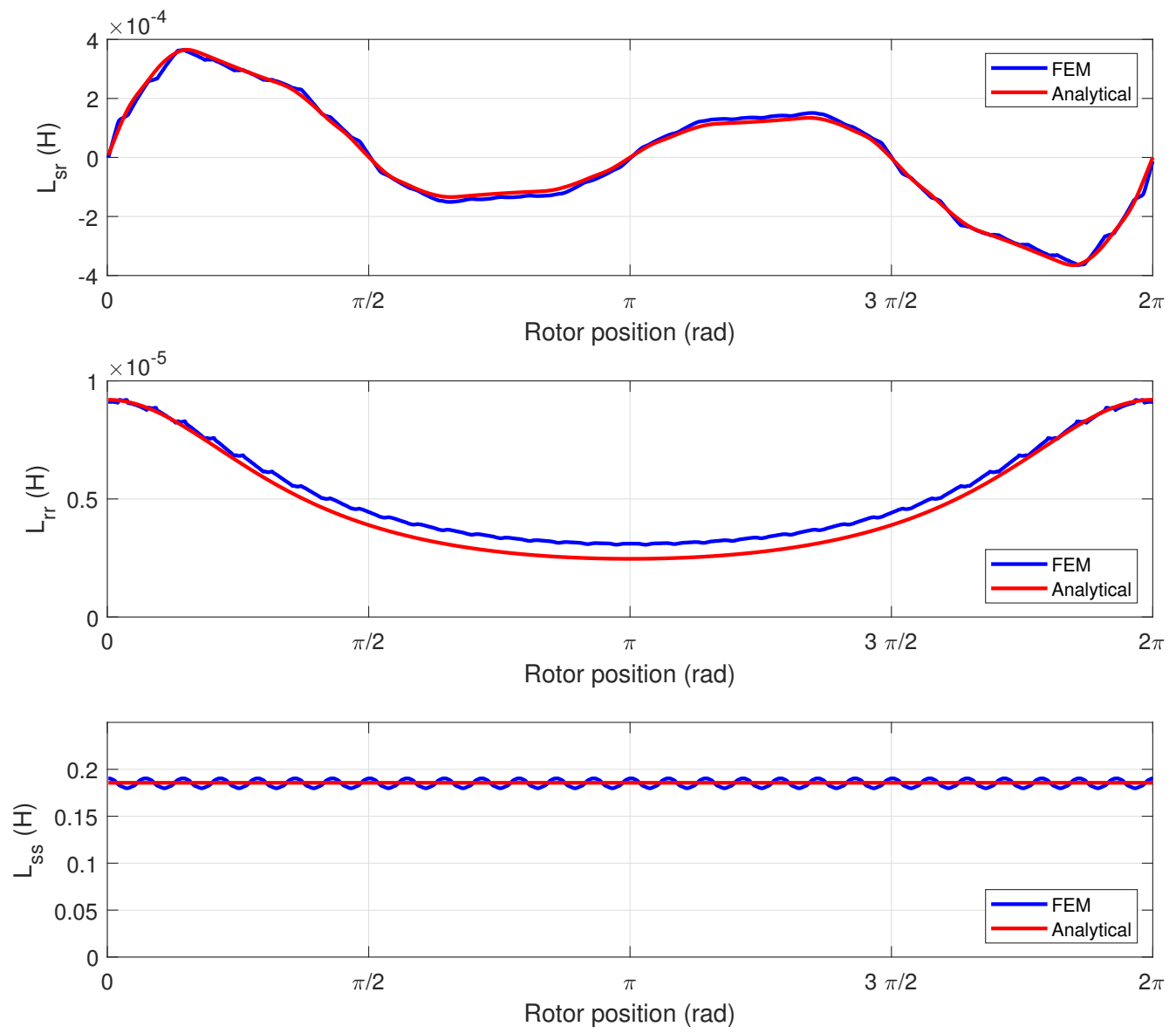

Figure 16: Comparison between the inductances obtained via FEM simulation (blue line) and with the proposed analytical method (red line), for the IM of Appendix A with a eccentricity level of $\delta_{s e}=0.6$ and $\delta_{d e}=0$ (static eccentricity only). Top: mutual inductance between the first stator phase and the first rotor loop. Middle: self-inductance of the first rotor loop. Bottom: self-inductance of the first stator phase. 

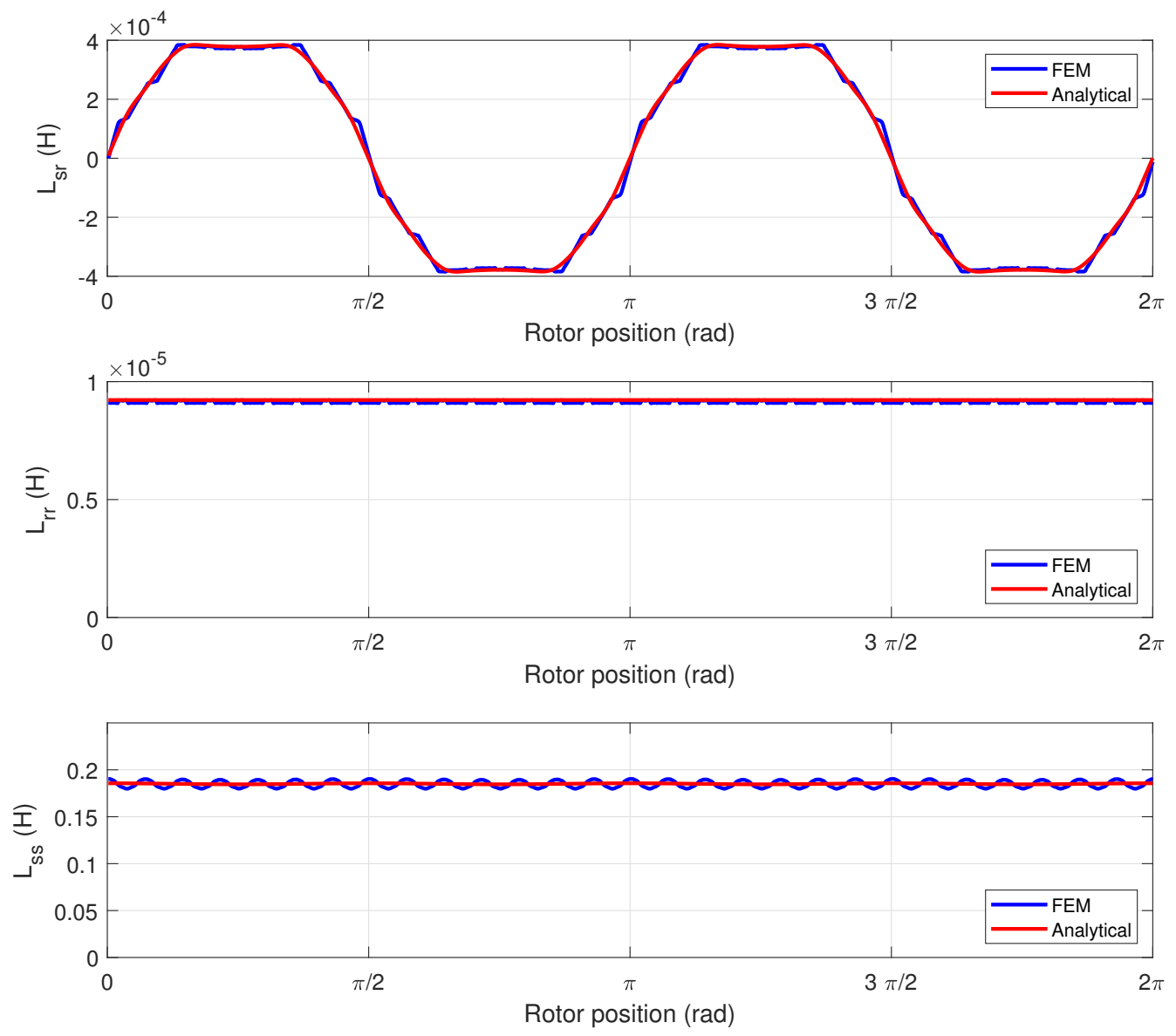

Figure 17: Comparison between the inductances obtained via FEM simulation (blue line) and with the proposed analytical method (red line), for the IM of Appendix A with a dynamic only eccentricity level of $\delta_{s e}=0$ and $\delta_{d e}=0.6$ (dynamic eccentricity only). Top: mutual inductance between the first stator phase and the first rotor loop. Middle: self-inductance of the first rotor loop. Bottom: self-inductance of the first stator phase.

To further assess the validity of the proposed method, the IM of Appendix A has been simulated with six different levels of static and dynamic eccentricity $\left(\delta_{s e}, \delta_{d e}\right)$, summarized in Table 3 . 
Table 3: Levels of static and dynamic eccentricity of the six simulated and experimental cases used in this work.

\begin{tabular}{cccc}
\hline Case N. & $\delta_{s e}$ & $\delta_{d e}$ & Remark \\
\hline 1 & 0.0 & 0.0 & Healthy machine \\
2 & 0.6 & 0.0 & Static eccentricity \\
3 & 0.4 & 0.2 & Mixed eccentricity \\
4 & 0.3 & 0.3 & Mixed eccentricity \\
5 & 0.2 & 0.4 & Mixed eccentricity \\
6 & 0.0 & 0.6 & Dynamic eccentricity \\
\hline
\end{tabular}

Fig. 18 compares the mutual inductance between the first stator phase and the first rotor loop obtained for the machine in Appendix A via FEM simulation (top) and with the proposed analytical method (bottom), corresponding to the six cases summarized in Table 3. Fig. 19 shows the same comparison for the self inductance of the first rotor loop, and Fig. 20 shows the same comparison for the self inductance of the first stator phase. In these figures the differences between the inductances computed with the FEM model and with the proposed method are also displayed. 

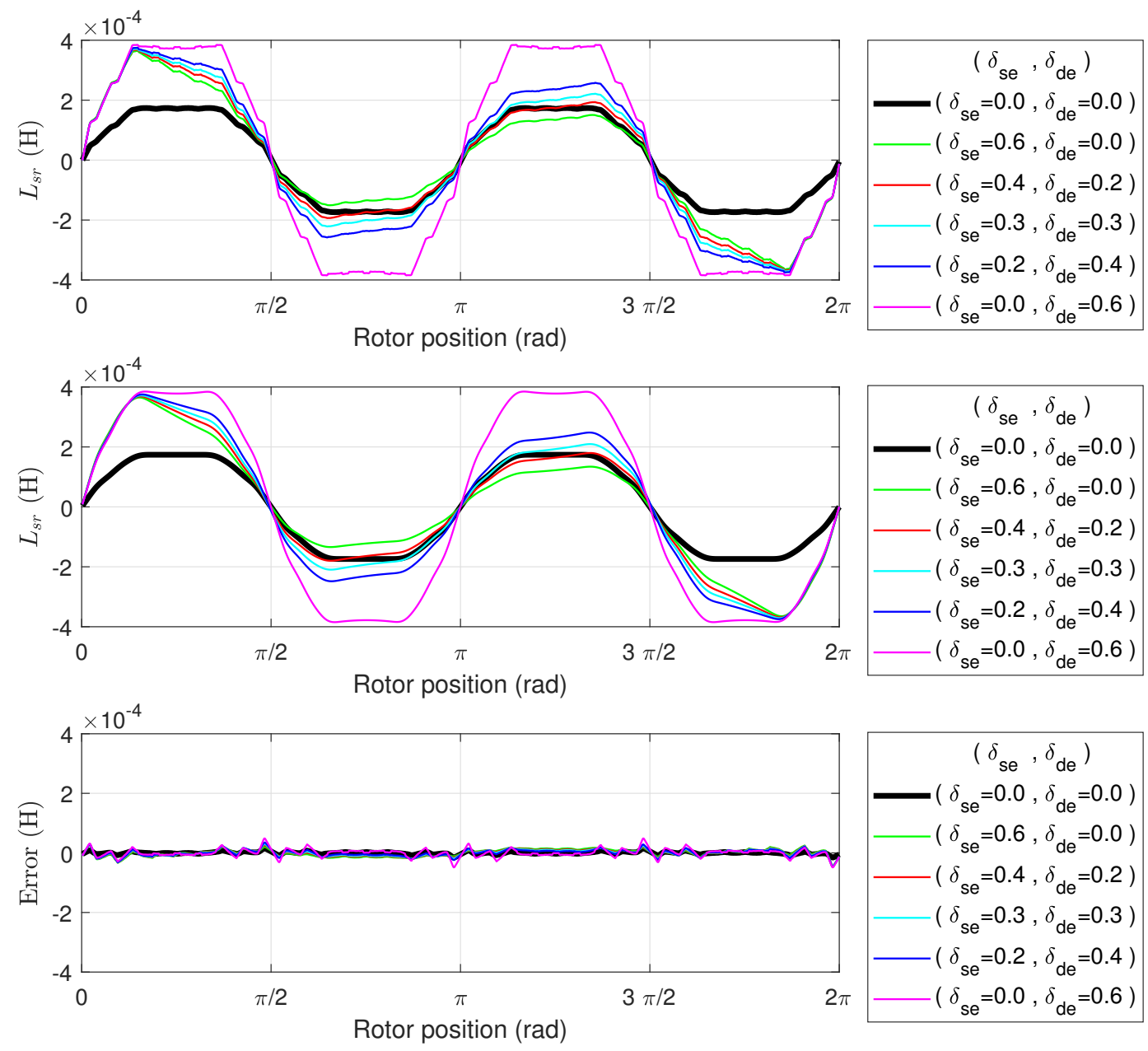

Figure 18: Mutual inductance vs. rotor position between the first stator phase and the first rotor loop of the machine in Appendix A, obtained via FEM simulation (top) and with the proposed analytical method (middle), and their differences (bottom). Six different levels of static and dynamic eccentricity $\left(\delta_{s e}, \delta_{d e}\right)$ have been plotted: $(0,0),(0.6,0),(0.4,0.2)$, $(0.3,0.3),(0.2,0.4)$ and $(0,0.6)$. 

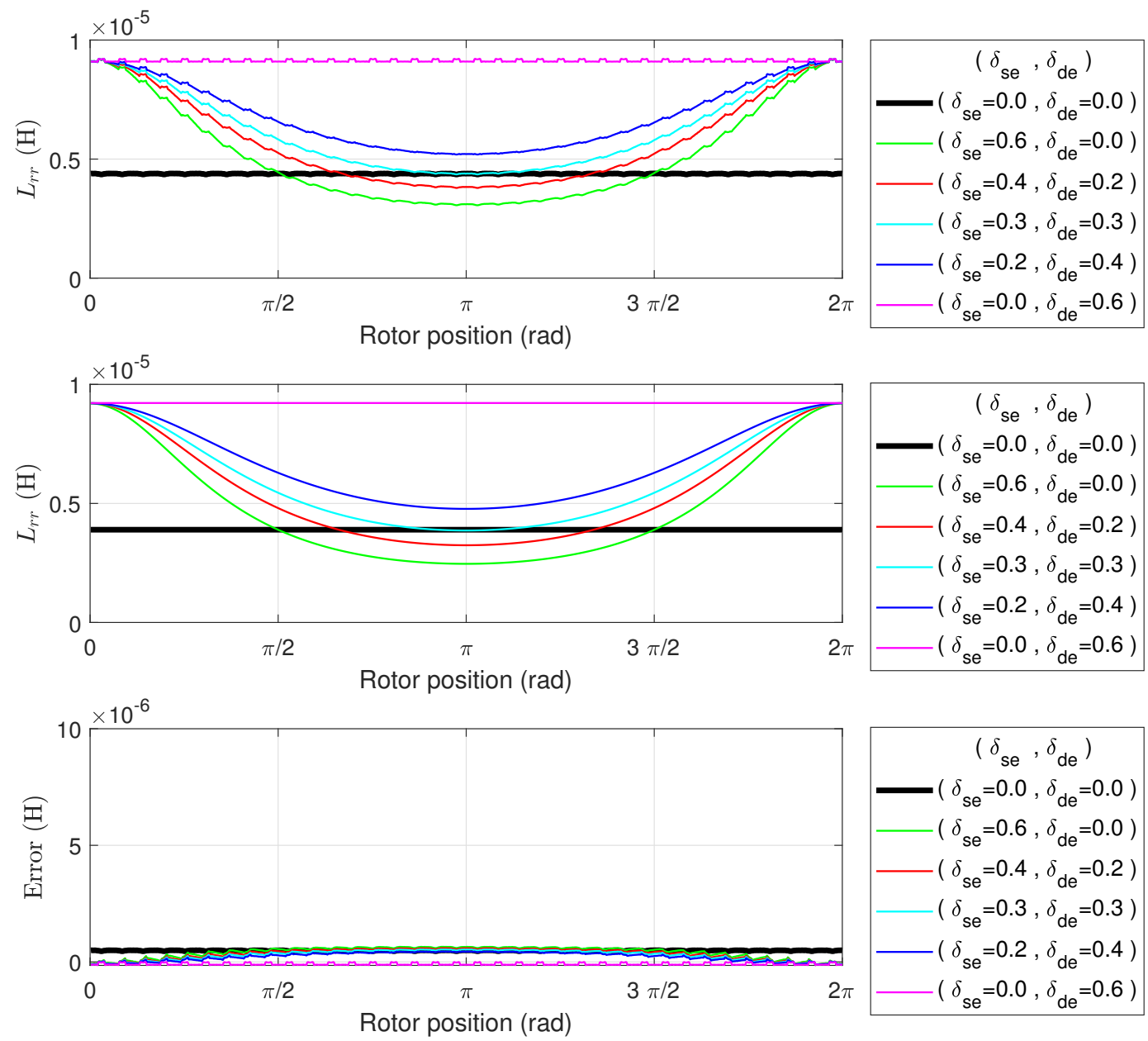

Figure 19: Self inductance vs. rotor position of the first rotor loop of the machine in Appendix A, obtained via FEM simulation (top) and with the proposed analytical method (middle), and their differences (bottom). Six different levels of static and dynamic eccentricity $\left(\delta_{s e}, \delta_{d e}\right)$ have been plotted: $(0,0),(0.6,0),(0.4,0.2),(0.3,0.3),(0.2,0.4)$ and $(0$, $0.6)$. 

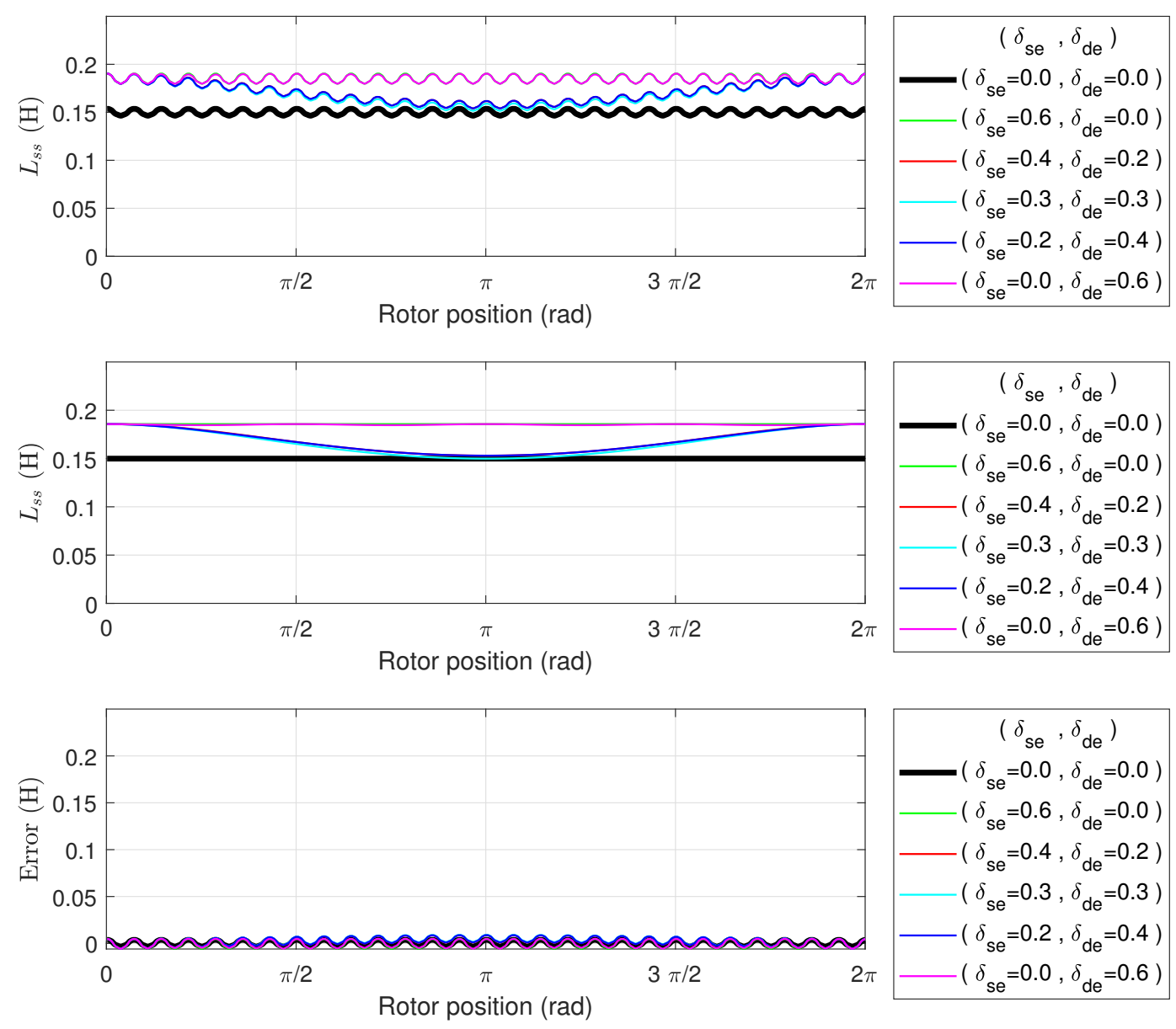

Figure 20: Self inductance vs. rotor position of the first stator phase of the machine in Appendix A, obtained via FEM simulation (top) and with the proposed analytical method (middle), and their differences (bottom). Six different levels of static and dynamic eccentricity $\left(\delta_{s e}, \delta_{d e}\right)$ have been plotted: $(0,0),(0.6,0),(0.4,0.2),(0.3,0.3),(0.2,0.4)$ and $(0,0.6)$.

Figures 18, 19 and 20 show a good agreement between the inductances calculated using the proposed analytical method, and with the FEM model, apart from slotting effects that are not included in the analytical model. To quantify this agreement, the normalized mean squared error (NMSE) has been computed for each case, as

$$
\operatorname{NMSE}\left(L_{F E M}, L_{\text {analytical }}\right)=\frac{\sum_{i}^{N}\left(L_{F E M}[i]-L_{\text {analytical }}[i]\right)^{2}}{\sum_{i}^{N}\left(L_{F E M}[i]\right)^{2}}
$$


where $L_{F E M}[i]$ and $L_{\text {analytical }}[i]$ are the $i t h$ elements of the two inductances matrices that are being compared, the first one obtained with the FEM method, and the second one with the proposed analytical method. The square root of the NMSE, expressed as a percentage value, has been summarized in Table 4 for the cases represented in Fig. 18, 19 and 20.

Table 4: Normalized root mean square error between the inductances obtained with the FEM model and with the proposed approach, as percentage values of the FEM model inductances.

\begin{tabular}{cccccc}
\hline Case N. & $\delta_{s e}$ & $\delta_{d e}$ & $\operatorname{Err}_{L_{s r}}$ & $\operatorname{Err}_{L_{r r}}$ & $\operatorname{Err}_{L_{s s}}$ \\
\hline 1 & 0.0 & 0.0 & $2.91 \%$ & $1.68 \%$ & $1.72 \%$ \\
2 & 0.6 & 0.0 & $6.24 \%$ & $8.57 \%$ & $2.05 \%$ \\
3 & 0.4 & 0.2 & $5.34 \%$ & $6.76 \%$ & $2.57 \%$ \\
4 & 0.3 & 0.3 & $4.85 \%$ & $5.57 \%$ & $2.71 \%$ \\
5 & 0.2 & 0.4 & $4.42 \%$ & $4.14 \%$ & $2.58 \%$ \\
6 & 0.0 & 0.6 & $4.25 \%$ & $1.12 \%$ & $1.94 \%$ \\
\hline
\end{tabular}

\subsection{Experimental Validation}

For the experimental validation of the suitability of the proposed analytical model of eccentric IM for diagnostic purposes, the motor whose characteristics are given in Appendix A has been endowed with an artificially provoked mixed eccentricity fault. For this purpose, each original bearing of the motor (see Fig.21.a) has been substituted by a new bearing (Fig. 21.d) with smaller outer diameter and greater inner diameter. Also two precision eccentric machined steel rings (Fig. 21.b and Fig. 21.c) have been used for adjusting the new bearing to the bearing housing (Fig. 21.b) and to the shaft (Fig. 21.c). The cylindrical surfaces of both rings are eccentric, $0.09 \mathrm{~mm}$ in the case of the outer ring b, and $0.09 \mathrm{~mm}$ in the case of the inner ring $\mathrm{c}$. Fig. 21.e shows the new assembly mounted on the shaft, obtaining in this way a rotor with a $30 \%$ of static eccentricity and a $30 \%$ of dynamic eccentricity. 


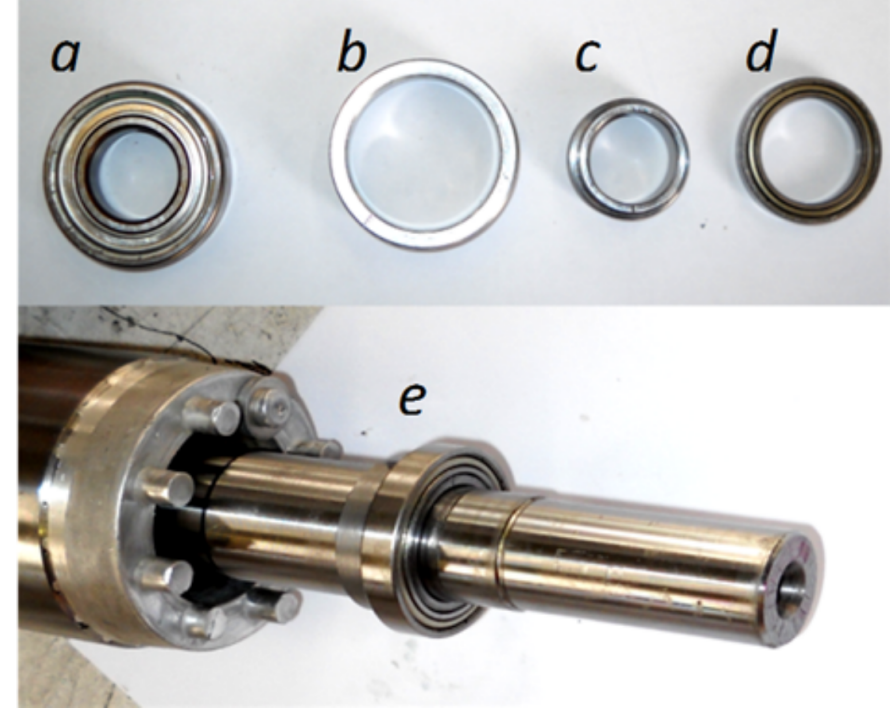

Figure 21: Rotor of the eccentric motor unit. Top, from left to right: a) original bearing, b) external and c) internal eccentric rings, and d) new bearing. Bottom: e) mounted unit on the shaft.

In the case of mixed eccentricity, it is well known in the technical literature [67] that this type of fault generates two different series of harmonics in the line current spectrum: a high frequency series of harmonics, which appear as sidebands around the principal slot harmonics, and a low frequency series of harmonics, which appear as sidebands around the fundamental component, at frequencies given by

$$
f_{M E}(s)=f_{1} \pm\left(k(1-s) f_{1} / p\right), \quad k=1,2,3 \ldots
$$

where $f_{1}$ is the power supply frequency, $s$ is the slip and $p$ is the number of pole pairs of the machine.

Focusing on the most dominant component of the series (53), obtained with $k=1$, a mixed eccentricity fault can be characterized by the presence in the stator current spectrum of components with frequencies given by:

$$
f_{M E}(s)=f_{1} \pm(1-s) f_{1} / p=f_{1} \pm f_{r}
$$

where $f_{r}$ is the rotational frequency of the motor. In the case of the tested 
motor, with $p=2$, (54) becomes

$$
f_{M E}(s)=f_{1} \pm(1-s) f_{1} / 2
$$

To verify the validity of the method proposed in this paper to reproduce the fault harmonics at frequencies given by (55), the motor of Appendix A has been tested at a speed of $1488 \mathrm{rpm}(s=(1500-1488) / 1500=0.008)$, under two different conditions: in healthy state, before mounting the eccentricity rings, and under faulty conditions, after mounting the eccentricity rings.

In both cases, one of the phase currents has been acquired, using the current clamp whose data is given in Appendix B, during an acquisition time of 10 seconds, with a sampling frequency of $5 \mathrm{kHz}$. The spectra of these currents are shown in Fig. 22 for the case of the motor in healthy condition (Fig. 22, top) and with the eccentricity rings mounted (Fig. 22, bottom). As expected from (55), two fault related harmonics appear in faulty conditions at frequencies $f_{M E}(0.008)=50 \pm(1-0.008) 50 / 2=[25.2 \mathrm{~Hz}, 74.8 \mathrm{~Hz}]$.

The motor of Appendix A has been simulated under the same conditions as the experimental test, $1488 \mathrm{rpm}$, both in healthy and faulty conditions, using the Simulink model given in [53]. In this model, the phases inductances matrix at each simulation time step is updated according to the rotor angular position, using the inductances matrix computed with (48), (49) and (50). The spectrum of the simulated stator phase current is given in Fig. 23, top, for the healthy condition, and in Fig. 23, bottom, for the eccentric fault condition. 

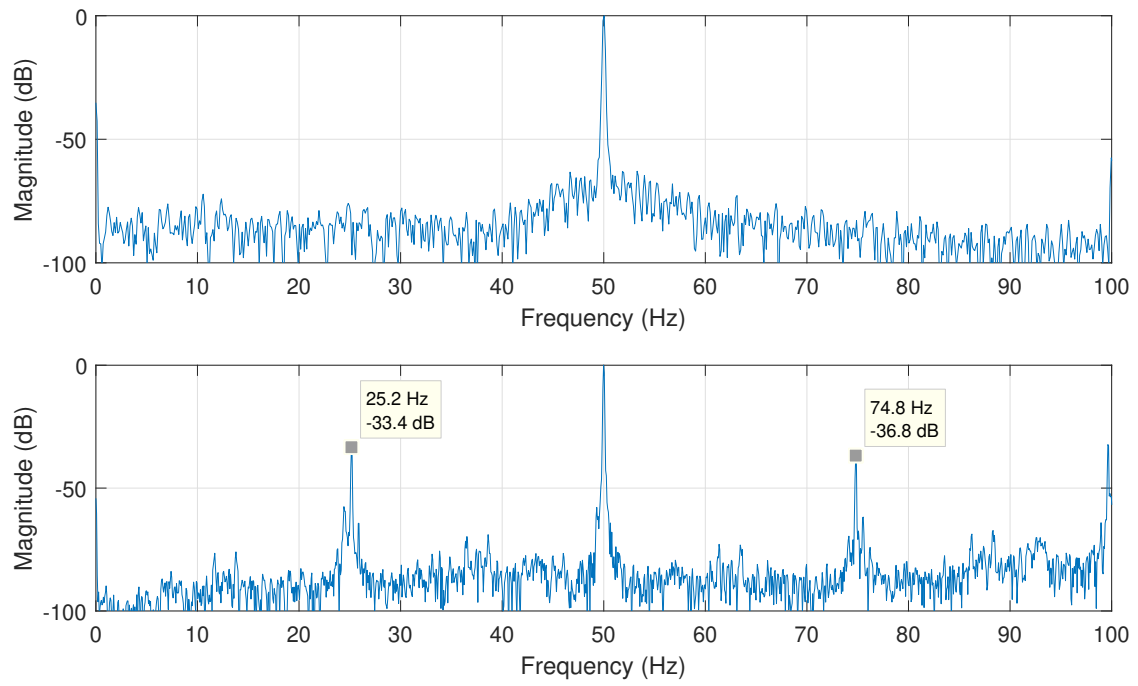

Figure 22: Spectrum of the experimental current of the motor Appendix A for the case of the motor in healthy condition (22, top) and with the eccentricity ring mounted (22, bottom). As expected from (55), two fault related harmonics appear in faulty conditions at frequencies $f_{M E}(0.008)=50 \pm(1-0.008) 50 / 2=[25.2 \mathrm{~Hz}, 74.8 \mathrm{~Hz}]$.
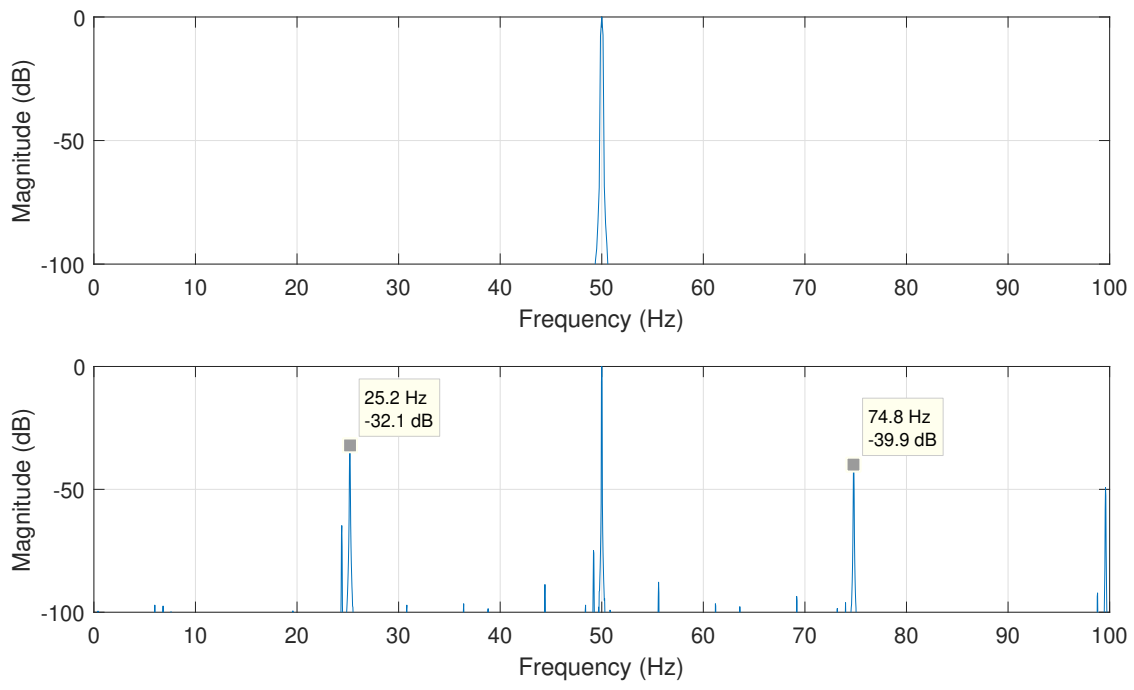

Figure 23: Spectrum of the stator phase current obtained from the simulation of the motor referenced in Appendix A in healthy condition (top), and with a mixed eccentricity of $\left(\delta_{s e}, \delta_{d e}\right)=(0.3,0.3)$ (bottom). These spectra show that the inductances matrix obtained with the method proposed in this paper is able to correctly reproduce the fault harmonics generated by a mixed eccentricity fault. 
As Fig. 23 shows, the inductances matrix obtained with the method proposed in this paper is able to correctly reproduce the fault harmonics generated by a mixed eccentricity fault (23, bottom), predicting accurately the frequencies of the fault components, and also giving a good approximation for their amplitudes.

\section{Conclusions}

This paper introduces a new analytical model suitable for simulating the IM under any type of eccentricity. The model is based on a novel approach for computing the phase inductances of the eccentric IM; this approach relies on two main novelties: first, an analytical expression for the yoke flux produced by a single conductor in an eccentric machine, as a function of the conductor and rotor position, and of the level of static and dynamic eccentric, has been obtained; and second, using this expression, a convolution-based procedure has been proposed for obtaining the inductances matrix, which gives the self and mutual inductances for every phases and rotor positions by a simple product in the spatial frequency domain, implemented with the FFT. The proposed convolution-based method enables to calculate the inductances matrix in few minutes, instead of hundreds of hours that will take this calculation using a FEM model. It is noticeable that, for a given level of dynamic and static eccentricity, the inductances matrix is valid, except for a scale factor, for any IM. The proposed method has been validated by comparing it with a FEM model, and with the results obtained from experimental tests with a commercial IM with a forced eccentricity fault.

\section{Appendix A. Commercial IM}

Three-phase induction machine. Rated characteristics: $P=1.1 \mathrm{~kW}$, $f=50 \mathrm{~Hz}, U=230 / 400 \mathrm{~V}, I=2.7 / 4.6 \mathrm{~A}, n=1410 \mathrm{r} / \mathrm{min}, \cos \varphi=0.8$.

Machine dimensions: Effective length of the magnetic core $=120 \mathrm{~mm}$, radius at the middle of the air gap $=54.1 \mathrm{~mm}$, air gap length $=0.28 \mathrm{~mm}$.

Stator: Three-phase winding, 36 slots, 78 wires/slot, winding pitch $=$ $7 / 9$, slot opening width $=2.1 \mathrm{~mm}$, phase resistance $7.68 \Omega$, end winding leakage $=2.3 \mathrm{mH}$.

Rotor: Squirrel-cage winding, 28 bars, slot opening width $=1.4 \mathrm{~mm}$, skew $=$ one slot pitch, bar resistance $=0.00202 \mathrm{~m} \Omega$, end winding leakage $=$ $2.45 \times 10^{-5} \mathrm{mH}$. 


\section{Appendix B. Current Clamp}

Chauvin Arnoux MN60, Nominal measuring scope: $100 \mathrm{~mA}-20 \mathrm{~A}$, ratio input/output: $1 \mathrm{~A} / 100 \mathrm{mV}$, intrinsic error: $\leq 2 \%+50 \mathrm{mV}$, frequency use: $400 \mathrm{~Hz}-10 \mathrm{kHz}$.

\section{Appendix C. Computer Features}

CPU: Intel Core i7-2600K CPU @ 3.40 GHZ RAM memory: 16 GB, Matlab Version: 9.4.0.813654 (R2018a).

\section{Appendix D. Inverse of the Air Gap Length in an Eccentric IM}

The expression of the inverse of the air gap length of an eccentric IM is obtained in this Appendix. The air gap length is given, from Fig. 2, by

$$
g(\varphi)=\left|\overline{O_{s} P_{S}}-\overline{O_{s} P_{R}}\right|=R_{s}-\left|\overline{O_{s} P_{R}}\right|
$$

The term $\overline{O_{s} P_{R}}$ can be expressed as a function of the rotor radius, $R_{r}$, as

$$
R_{r}^{2}=\left|\overline{O_{s} P_{R}}\right|^{2}+\left(g_{0} \cdot \delta_{r}\right)^{2}-2\left|\overline{O_{s} P_{R}}\right| \cdot g_{0} \cdot \delta_{r} \cdot \cos (\varphi)
$$

that is,

$$
\left|\overline{O_{s} P_{R}}\right|=\frac{2 g_{0} \cdot \delta_{r} \cdot \cos (\varphi) \pm \sqrt{\left(2 g_{0} \cdot \delta_{r} \cdot \cos (\varphi)\right)^{2}-4\left(g_{0}^{2} \cdot \delta_{r}{ }^{2}-R_{r}^{2}\right)}}{2}
$$

and, substituting (D.3) in (D.1) gives

$$
g(\varphi)=R_{s}-g_{0} \cdot \delta_{r} \cos (\varphi) \pm \sqrt{\left(g_{0} \cdot \delta_{r} \cdot \cos (\varphi)\right)^{2}-g_{0}^{2} \cdot \delta_{r}{ }^{2}+R_{r}^{2}}
$$

Assuming that the radius of the rotor is much greater than the air gap length, $R_{r}>>g_{0} \cdot \delta_{r}$, then (D.4) becomes

$$
\left.g(\varphi) \approx R_{s}-R_{r}-g_{0} \cdot \delta_{r} \cos (\varphi)\right) \approx g_{0}\left(1-\delta_{r} \cos (\varphi)\right)
$$

From (D.5), the air gap length at any given angular coordinate $\varphi$ can be approximated by a function of the rotor center coordinates $\delta_{r}$ and $\Theta_{r}$ as

$$
g\left(\varphi, \Theta_{r}, \delta_{r}\right) \approx g_{0} \cdot\left(1-\delta_{r} \cdot \cos \left(\varphi-\Theta_{r}\right)\right)
$$


For computing the phase inductances, it is needed the inverse of the air gap function (D.6) to obtain the permeance function of the machine:

$$
g\left(\varphi, \Theta_{r}, \delta_{r}\right)^{-1}=g_{0}^{-1} \cdot \frac{1}{\left(1-\delta_{r} \cdot \cos \left(\varphi-\Theta_{r}\right)\right)}
$$

Neglecting the variations of the mean air gap radius (26), the function given by (D.7) can be expressed as the series [62]

$\left.\frac{1}{1-\delta_{r} \cdot \cos \left(\varphi-\Theta_{r}\right)}=\frac{1}{\sqrt{1-\delta_{r}{ }^{2}}}+2 \sum_{m=1}^{\infty}\left[\left(\frac{1-\sqrt{1-\delta_{r}{ }^{2}}}{\sqrt{1-\delta_{r}{ }^{2}}}\right)^{m} \cos \left(m\left(\varphi-\Theta_{r}\right)\right)\right)\right]$

Applying (D.8) to (D.7) gives finally

$$
g\left(\varphi, \Theta_{r}, \delta_{r}\right)^{-1}=g_{0}^{-1} \cdot\left(A_{0}+\sum_{m=1}^{n_{t}} A_{m} \cdot \cos \left(m\left(\varphi-\Theta_{r}\right)\right)\right)
$$

where

$$
A_{0}=\frac{1}{\sqrt{1-\delta_{r}^{2}}} \quad A_{m}=2\left(\frac{1-\sqrt{1-\delta_{r}^{2}}}{\sqrt{1-\delta_{r}^{2}}}\right)^{m} \quad m=1 \ldots n_{t}
$$

\section{Acknowledgements}

This work was supported by the Spanish "Ministerio de Ciencia, Innovación y Universidades (MCIU)", the "Agencia Estatal de Investigación $(\mathrm{AEI}) "$ and the "Fondo Europeo de Desarrollo Regional (FEDER)" in the framework of the "Proyectos I+D+i - Retos Investigación 2018", project reference RTI2018-102175-B-I00 (MCIU/AEI/FEDER, UE).

\section{References}

[1] Y. Liu, A. M. Bazzi, A review and comparison of fault detection and diagnosis methods for squirrel-cage induction motors: State of the art, ISA Trans. 70 (2017) 400-409. 
[2] A. Balasubramanian, R. Muthu, Model Based Fault Detection and Diagnosis of Doubly Fed Induction Generators - A Review, Energy Procedia 117 (2017) 935-942.

[3] A. Bellini, F. Filippetti, C. Tassoni, G.-A. Capolino, Advances in Diagnostic Techniques for Induction Machines, IEEE Trans. Ind. Electron. 55 (2008) 4109-4126.

[4] I. Culbert, J. Letal, Signature Analysis for Online Motor Diagnostics: Early Detection of Rotating Machine Problems Prior to Failure, IEEE Ind. Appl. Mag. 23 (2017) 76-81.

[5] M. Manohar, E. Koley, S. Ghosh, Enhancing resilience of PV-fed microgrid by improved relaying and differentiating between inverter faults and distribution line faults, Int. J. Electr. Power Energy Syst. 108 (2019) 271-279.

[6] X. Xia, J. Zhou, C. Li, W. Zhu, A novel method for fault diagnosis of hydro generator based on NOFRFs, Int. J. Electr. Power Energy Syst. 71 (2015) 60-67.

[7] B. Zhao, M. Yang, H. Diao, B. An, Y. Zhao, Y. Zhang, A novel approach to transformer fault diagnosis using IDM and naive credal classifier, Int. J. Electr. Power Energy Syst. 105 (2019) 846-855.

[8] G. Rigatos, P. Siano, Power transformers' condition monitoring using neural modeling and the local statistical approach to fault diagnosis, Int. J. Electr. Power Energy Syst. 80 (2016) 150-159.

[9] Y. Zhang, Y. Zhang, F. Wen, C. Y. Chung, C.-L. Tseng, X. Zhang, F. Zeng, Y. Yuan, A fuzzy Petri net based approach for fault diagnosis in power systems considering temporal constraints, Int. J. Electr. Power Energy Syst. 78 (2016) 215-224.

[10] M. Farshad, Detection and classification of internal faults in bipolar HVDC transmission lines based on K-means data description method, Int. J. Electr. Power Energy Syst. 104 (2019) 615-625.

[11] M. M. Morato, D. J. Regner, P. R. Mendes, J. E. Normey-Rico, C. Bordons, Fault Analysis, Detection and Estimation for a Microgrid via 
H2/HLPV Observers, Int. J. Electr. Power Energy Syst. 105 (2019) $823-845$.

[12] K. Yahia, M. Sahraoui, A. J. M. Cardoso, A. Ghoggal, The Use of a Modified Prony's Method to Detect the Airgap-Eccentricity Occurrence in Induction Motors, IEEE Trans. Ind. Appl. 52 (2016) 3869-3877.

[13] E. H. El Bouchikhi, V. Choqueuse, M. Benbouzid, Induction machine faults detection using stator current parametric spectral estimation, Mech. Syst. Signal Process. 52-53 (2015) 447-464.

[14] C. Morales-Perez, J. Rangel-Magdaleno, H. Peregrina-Barreto, J. P. Amezquita-Sanchez, M. Valtierra-Rodriguez, Incipient Broken Rotor Bar Detection in Induction Motors Using Vibration Signals and the Orthogonal Matching Pursuit Algorithm, IEEE Trans. Instrum. Meas. 67 (2018) 2058-2068.

[15] J. Faiz, M. Ojaghi, Instantaneous-Power Harmonics as Indexes for Mixed Eccentricity Fault in Mains-Fed and Open/Closed-Loop DriveConnected Squirrel-Cage Induction Motors, IEEE Trans. Ind. Electron. 56 (2009) 4718-4726.

[16] M. E. K. Oumaamar, Y. Maouche, M. Boucherma, A. Khezzar, Static air-gap eccentricity fault diagnosis using rotor slot harmonics in line neutral voltage of three-phase squirrel cage induction motor, Mech. Syst. Signal Process. 84 (2017) 584-597.

[17] E. Resendiz-Ochoa, R. A. Osornio-Rios, J. P. Benitez-Rangel, R. De J. Romero-Troncoso, L. A. Morales-Hernandez, Induction Motor Failure Analysis: An Automatic Methodology Based on Infrared Imaging, IEEE Access 6 (2018) 76993-77003.

[18] G. Mirzaeva, K. I. Saad, Advanced Diagnosis of Stator Turn-to-Turn Faults and Static Eccentricity in Induction Motors Based on Internal Flux Measurement, IEEE Trans. Ind. Appl. 54 (2018) 3961-3970.

[19] B. Parvathi Sangeetha and S. Hemamalini, Rational-dilation wavelet transform based torque estimation from acoustic signals for fault diagnosis in a three-phase induction motor, IEEE Transactions on Industrial Informatics 15 (2019) 3492-3501. 
[20] A. Naha, A. K. Samanta, A. Routray, A. K. Deb, Low Complexity Motor Current Signature Analysis Using Sub-Nyquist Strategy With Reduced Data Length, IEEE Trans. Instrum. Meas. 66 (2017) 3249-3259.

[21] M. Y. Kaikaa, M. Hadjami, A. Khezzar, Effects of the simultaneous presence of static eccentricity and broken rotor bars on the stator current of induction machine, IEEE Trans. Ind. Electron. 61 (2014) 2452-2463.

[22] G. Singh, V. Naikan, Detection of half broken rotor bar fault in VFD driven induction motor drive using motor square current MUSIC analysis, Mech. Syst. Signal Process. 110 (2018) 333-348.

[23] A. Singh, B. Grant, R. DeFour, C. Sharma, S. Bahadoorsingh, A review of induction motor fault modeling, Electr. Power Syst. Res. 133 (2016) 191-197.

[24] P. Gangsar, R. Tiwari, A support vector machine based fault diagnostics of Induction motors for practical situation of multi-sensor limited data case, Meas. J. Int. Meas. Confed. 135 (2019) 694-711.

[25] M. Ojaghi, R. Aghmasheh, M. Sabouri, Model-based exact technique to identify type and degree of eccentricity faults in induction motors, IET Electr. Power Appl. 10 (2016) 706-713.

[26] A. A. Salah, D. G. Dorrell, Y. Guo, A Review of the Monitoring and Damping Unbalanced Magnetic Pull in Induction Machines Due to Rotor Eccentricity, IEEE Trans. Ind. Appl. 55 (2019) 2569 - 2580.

[27] J. Faiz, S. M. M. Moosavi, Detection of mixed eccentricity fault in doubly-fed induction generator based on reactive power spectrum, IET Electr. Power Appl. 11 (2017) 1076-1084.

[28] P. Naderi, F. Fallahi, Eccentricity fault diagnosis in three-phase-woundrotor induction machine using numerical discrete modeling method, Int. J. Numer. Model. Electron. Networks, Devices Fields 29 (2016) 982-997.

[29] J. Faiz, S. Moosavi, Eccentricity fault detection - From induction machines to DFIG - A review, Renew. Sustain. Energy Rev. 55 (2016) 169-179. 
[30] B. Silwal, P. Rasilo, L. Perkkio, A. Hannukainen, T. Eirola, A. Arkkio, Numerical Analysis of the Power Balance of an Electrical Machine With Rotor Eccentricity, IEEE Trans. Magn. 52 (2016) 1-4.

[31] Y. Yao, Y. Li, Q. Yin, A novel method based on self-sensing motor drive system for misalignment detection, Mech. Syst. Signal Process. 116 (2019) 217-229.

[32] Y. Zhou, X. Bao, C. Di, L. Wang, Analysis of Dynamic Unbalanced Magnetic Pull in Induction Motor With Dynamic Eccentricity During Starting Period, IEEE Trans. Magn. 52 (2016) 1-4.

[33] Chong Di, Xiaohua Bao, Hanfeng Wang, Qiang Lv, Yigang He, Modeling and Analysis of Unbalanced Magnetic Pull in Cage Induction Motors With Curved Dynamic Eccentricity, IEEE Trans. Magn. 51 (2015) 1-7.

[34] D. G. Dorrell, Sources and Characteristics of Unbalanced Magnetic Pull in Three-Phase Cage Induction Motors With Axial-Varying Rotor Eccentricity, IEEE Trans. Ind. Appl. 47 (2011) 12-24.

[35] X. Xu, Q. Han, F. Chu, A general electromagnetic excitation model for electrical machines considering the magnetic saturation and rub impact, J. Sound Vib. 416 (2018) 154-171.

[36] J. Faiz, B. M. Ebrahimi, M. B. B. Sharifian, Finite Element Transient Analysis of an On-Load Three-Phase Squirrel-Cage Induction Motor with Static Eccentricity, Electromagnetics 27 (2007) 207-227.

[37] M. DeBortoli, S. Salon, D. Burow, C. Slavik, Effects of rotor eccentricity and parallel windings on induction machine behavior: a study using finite element analysis, IEEE Trans. Magn. 29 (1993) 1676-1682.

[38] J. Faiz, B. Ebrahimi, B. Akin, H. Toliyat, Comprehensive Eccentricity Fault Diagnosis in Induction Motors Using Finite Element Method, IEEE Trans. Magn. 45 (2009) 1764-1767.

[39] J. Martinez, A. Belahcen, J. Detoni, A. Arkkio, A 2D FEM analysis of electromechanical signatures in induction motors under dynamic eccentricity, Int. J. Numer. Model. Electron. Networks, Devices Fields 27 (2014) 555-571. 
[40] M. Thiele, G. Heins, Computationally Efficient Method for Identifying Manufacturing Induced Rotor and Stator Misalignment in Permanent Magnet Brushless Machines, IEEE Trans. Ind. Appl. 52 (2016) 30333040 .

[41] E. Ajily, M. Ardebili, K. Abbaszadeh, Magnet Defect and Rotor Eccentricity Modeling in Axial-Flux Permanent-Magnet Machines via 3-D Field Reconstruction Method, IEEE Trans. Energy Convers. 31 (2016) 486-495.

[42] H. Mahmoud, N. Bianchi, Eccentricity in Synchronous Reluctance Motors - Part I: Analytical and Finite-Element Models, IEEE Trans. Energy Convers. 30 (2015) 745-753.

[43] T. Ilamparithi, S. Nandi, Comparison of results for eccentric cage induction motor using Finite Element method and Modified Winding Function Approach, in: 2010 Jt. Int. Conf. Power Electron. Drives Energy Syst. 2010 Power India, IEEE, 2010, pp. 1-7.

[44] J. Faiz, M. Ghasemi-Bijan, B. Mahdi Ebrahimi, Modeling and Diagnosing Eccentricity Fault Using Three-dimensional Magnetic Equivalent Circuit Model of Three-phase Squirrel-cage Induction Motor, Electr. Power Components Syst. 43 (2015) 1246-1256.

[45] H. Garg, R. Dahiya, Current signature analysis and its application in the condition monitoring of wind turbine for rotor faults, Energy Syst. 8 (2017) 495-510.

[46] X. Bao, Z. Cheng, C. Di, Current analysis of large submersible motor under curved eccentricity by multi-loop method, Int. J. Appl. Electromagn. Mech. 53 (2017) 63-76.

[47] B. Rajalakshmi Samaga, K. Vittal, Comprehensive study of mixed eccentricity fault diagnosis in induction motors using signature analysis, Int. J. Electr. Power Energy Syst. 35 (2012) 180-185.

[48] A. Ghoggal, S. Zouzou, H. Razik, M. Sahraoui, A. Khezzar, An improved model of induction motors for diagnosis purposes - Slot skewing effect and air-gap eccentricity faults, Energy Convers. Manag. 50 (2009) 13361347 . 
[49] J. Faiz, M. Ojaghi, Unified winding function approach for dynamic simulation of different kinds of eccentricity faults in cage induction machines, IET Electr. Power Appl. 3 (2009) 461.

[50] H. A. Toliyat, T. A. Lipo, J. C. White, Analysis of a concentrated winding induction machine for adjustable speed drive applications. I. Motor analysis, IEEE Trans. Energy Convers. 6 (1991) 679-683.

[51] A. Ghoggal, S. E. Zouzou, H. Razik, M. Sahraoui, A. Hadri-Hamida, Application of the convolution theorem for the modeling of saturated induction motors, in: IECON 2010 - 36th Annu. Conf. IEEE Ind. Electron. Soc., IEEE, 2010, pp. 772-777.

[52] A. Ghoggal, M. Sahraoui, S. E. Zouzou, H. Razik, A Fast Inductance Computation Devoted to the Modeling of Healthy, Eccentric, and Saturated Induction Motors, Electr. Power Components Syst. 41 (2013) $1002-1022$.

[53] A. Sapena-Bano, J. Martinez-Roman, R. Puche-Panadero, M. PinedaSanchez, J. Perez-Cruz, M. Riera-Guasp, Induction machine model with space harmonics for fault diagnosis based on the convolution theorem, Int. J. Electr. Power Energy Syst. 100 (2018) 463-481.

[54] Y. Horen, P. Strajnikov, A. Kuperman, Simple mechanical parameters identification of induction machine using voltage sensor only, Energy Conversion and Management 92 (2015) $60-66$.

[55] M. Pineda-Sanchez, R. Puche-Panadero, J. Martinez-Roman, A. Sapena-Bano, M. Riera-Guasp, J. Perez-Cruz, Partial Inductance Model of Induction Machines for Fault Diagnosis, Sensors 18 (2018) 2340.

[56] M. Ikeda, T. Hiyama, Simulation Studies of the Transients of SquirrelCcage Induction Motors, IEEE Trans. Energy Convers. 22 (2007) 233239.

[57] J. Staszak, Determination of slot leakage inductance for three-phase induction motor winding using an analytical method, Archives of Electrical Engineering 62 (2013) 569 - 591. 
[58] G. Joksimovic, M. Durovic, J. Penman, N. Arthur, Dynamic simulation of dynamic eccentricity in induction machines-winding function approach, IEEE Trans. Energy Convers. 15 (2000) 143-148.

[59] S. Nandi, R. Bharadwaj, H. Toliyat, Performance analysis of a threephase induction motor under mixed eccentricity condition, IEEE Trans. Energy Convers. 17 (2002) 392-399.

[60] J. Faiz, I. Tabatabaei, Extension of winding function theory for nonuniform air gap in electric machinery, IEEE Trans. Magn. 38 (2002) 36543657 .

[61] G. Bossio, C. De Angelo, J. Solsona, G. Garcia, M. I. Valla, A 2-D model of the induction machine: an extension of the modified winding function approach, Energy Conversion, IEEE Trans. 19 (2004) 144-150.

[62] J. Faiz, I. Ardekanei, H. Toliyat, An evaluation of inductances of a squirrel-cage induction motor under mixed eccentric conditions, IEEE Trans. Energy Convers. 18 (2003) 252-258.

[63] B. Hague, The principles of electromagnetism applied to electrical machines, Dover, 1962.

[64] B. Heller, V. Hamata, Harmonic field effects in induction machines, Elsevier Science \& Technology, 1977.

[65] X. Li, Q. Wu, S. Nandi, Performance Analysis of a Three-Phase Induction Machine With Inclined Static Eccentricity, IEEE Trans. Ind. Appl. 43 (2007) 531-541.

[66] D. Meeker, Finite Element Method Magnetics. User's Manual. Version 4.0, 2004.

[67] S. Nandi, R. M. Bharadwaj, H. A. Toliyat, Performance Analysis of a Three-Phase Induction Motor Under Mixed Eccentricity Condition, IEEE Trans. Energy Convers. 17 (2002) 392-399. 